\title{
Rapid vigilance and episodic memory decrements in COVID-I9 survivors
}

\author{
(1)Sijia Zhao,' Kengo Shibata, ${ }^{2}$ Peter J. Hellyer, ${ }^{3,4}$ @W William Trender, ${ }^{3}$ (I) Sanjay Manohar, ${ }^{1,2}$ \\ (1)Adam Hampshire ${ }^{3}$ and (1) Masud Husain ${ }^{1,2}$
}

Recent studies indicate that COVID-19 infection can lead to serious neurological consequences in a small percentage of individuals. However, in the months following acute illness, many more suffer from fatigue, low motivation, disturbed mood, poor sleep and cognitive symptoms, colloquially referred to as 'brain fog'. But what about individuals who had asymptomatic to moderate COVID-19 and reported no concerns after recovering from COVID-19? Here, we examined a wide range of cognitive functions critical for daily life (including sustained attention, memory, motor control, planning, semantic reasoning, mental rotation and spatialvisual attention) in people who had previously suffered from COVID-19 but were not significantly different from a control group on self-reported fatigue, forgetfulness, sleep abnormality, motivation, depression, anxiety and personality profile. Reassuringly, COVID-19 survivors performed well in most abilities tested, including working memory, executive function, planning and mental rotation. However, they displayed significantly worse episodic memory (up to 6 months post-infection) and greater decline in vigilance with time on task (for up to 9 months). Overall, the results show that specific chronic cognitive changes following COVID-19 are evident on objective testing even amongst those who do not report a greater symptom burden. Importantly, in the sample tested here, these were not significantly different from normal after 6-9 months, demonstrating evidence of recovery over time.

1 Department of Experimental Psychology, University of Oxford, Oxford OX1 3PH, UK

2 Nuffield Department of Clinical Neurosciences, University of Oxford, Oxford OX3 9DU, UK

3 Department of Brain Sciences, Imperial College London, 926, Sir Michael Uren Hub, 86 Wood Lane, London W12 0BZ, UK

4 Institute of Psychiatry, Psychology and Neuroscience, King's College London, London, UK

Correspondence to: Sijia Zhao

Department of Experimental Psychology

University of Oxford

Oxford OX1 3PH, UK

E-mail: sijia.zhao@psy.ox.ac.uk

Keywords: COVID-19; long-COVID; cognitive deficits; sustained attention; memory

Abbreviations: $\mathrm{AMI}=$ Apathy Motivation Index; $\mathrm{BF}=$ Bayesian Factor; $\mathrm{BFI}-\mathrm{S}=$ Short Big Five Inventory; $\mathrm{CFQ}=\mathrm{Cognitive}$ Failures Questionnaire; COVID-19=Coronavirus disease 2019 caused by the Coronavirus SARS-CoV-2; GLM=generalized linear model; GRIT-S = Short Grit Scale; HADS = Hospital Anxiety and Depression Scale; LMM=linear mixed-effect model; $\mathrm{NFI}=$ Neurological Fatigue Index; PCA = Principal Component Analysis; SD = standard deviation; SES = socioeconomic status 


\section{Attention declines rapidly over minutes in people who had COVID-19}
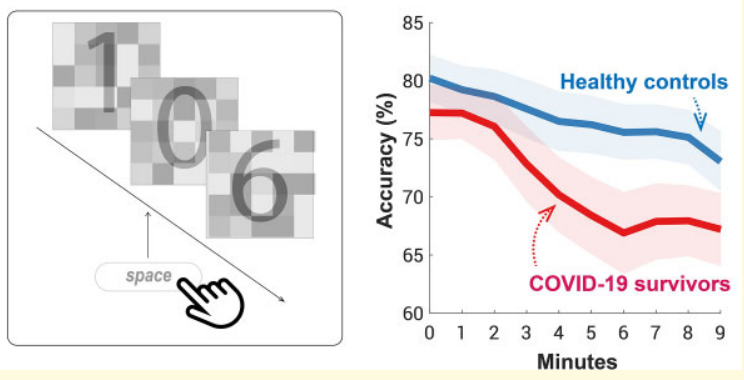

\section{Introduction}

People who survive COVID-19 infection present a significantly higher risk of major neurological and psychiatric conditions, particularly if they were hospitalized. ${ }^{1-3}$ These include acute cerebrovascular events such as ischaemic stroke and intracerebral haemorrhage. ${ }^{4}$ In addition to severe neurological conditions, there can also be more chronic, longer-term consequences such as fatigue, low motivation, disturbed mood and poor sleep-all commonly reported symptoms amongst survivors, the so-called long-COVID (see recent review ${ }^{5}$ ).

Whilst many studies employ questionnaires reliant on patients' subjective self-reports, recent investigations have begun to document a wide range of post-COVID-19 cognitive deficits using objective cognitive testing of inpatients. Particular impairments were found in attention, ${ }^{6-12}$ memory ${ }^{6-8,10-13}$ and executive functions. ${ }^{10-12,14-16}$ More recently, using ${ }^{18} \mathrm{~F}$-FDG PET, it has been demonstrated that in the most severely affected patients, the degree of cognitive impairment was accompanied by frontoparietal hypometabolism. ${ }^{10,11}$

Understandably, most of these small-scale reports have, to date, predominantly focused on symptomatic, hospitalized patients (however $\operatorname{se}^{17}$ ). But what about individuals who have not been hospitalized and do not report any ongoing symptoms after recovery? Do they suffer cognitive deficits that they are not aware of? Here, we examined people who had previously contracted COVID-19 but were not significantly different from a control group with respect to self-reported fatigue, forgetfulness, sleep abnormality, motivation, depression, anxiety or personality profile. In total, 135 participants were tested on a series of 12 online cognitive tasks encompassing a wide range of cognitive functions critical for daily life, including the ability to sustain attention, memory, motor control, speed of response, planning, semantic reasoning, mental rotation and spatial-visual attention. In all of these functions, COVID-19 survivors showed the same initial baseline performance. However, after only two minutes on an attentionally demanding

task, there was a significantly larger decline in perceptual sensitivity, despite reporting the same levels of fatigue compared to healthy controls. Subsequent testing also revealed a significantly larger episodic memory decrement. These results demonstrated that chronic cognitive reductions following COVID-19 are evident upon objective testing even in people who do not report long-COVID symptoms.

\section{Materials and methods}

\section{Participants}

One hundred and fifty-five participants $\{59$ females, mean age 28.6 years [standard deviation (SD) 9.7]\} were recruited from the Prolific online recruitment platform (http://www. prolific.co). All participants were naïve about the aim of this study; the study was advertised as 'a brain game' testing how well people could perform. Sixty-four people had contracted COVID-19, whilst 91 reported that they had not. Although none had received treatment in an intensive care unit or were seeking post-COVID care at the time the study was conducted, three participants had been hospitalized for COVID-19, seven participants had severe COVID-19 symptoms (i.e. their COVID-19 symptoms largely reduced their ability to carry-out day-to-day activities) and two participants had severe long-COVID symptoms. In total, 11 participants were excluded. In addition, a follow-up survey showed that eight control participants later discovered that they contracted COVID-19 before via antigen detection test; although we did not find any difference in behavioural performance between them the rest of the control group, they were also excluded from further analysis.

In all, 136 participants were included in further analysis (COVID $n=53$, Control $n=83$, see Fig. 1 for study population flow and Table 1 for demographics). There was no significant group difference in gender $\left[\chi^{2}(1, N=54)=0.5, P=0.5\right)$ or age $t(134)=-0.6, P=0.5$, Bayesian Factor $(\mathrm{BF})=4.4]$.

All participants were required to complete the experiments on the Chrome browser on a desktop with a keyboard and mouse. 


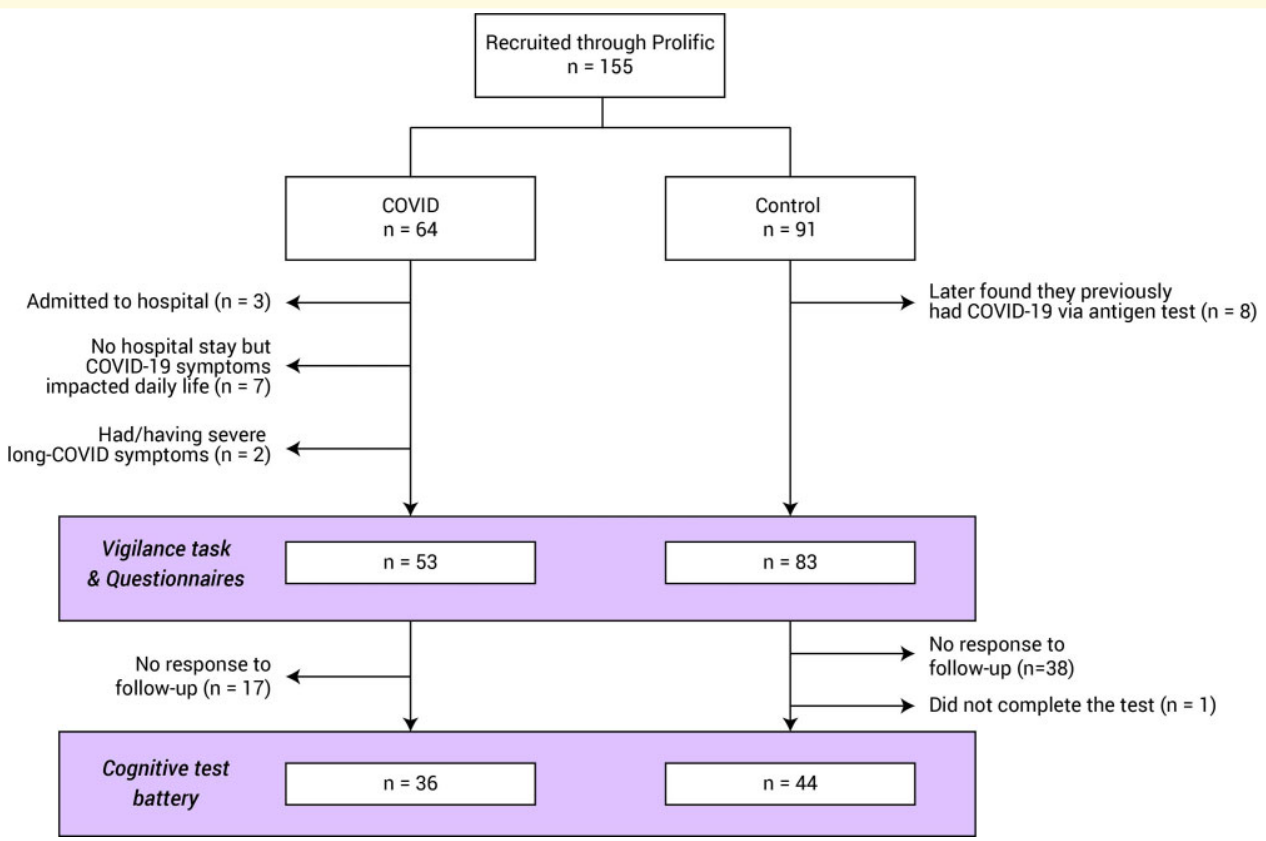

Figure I Study population flow chart. Number of participants eligible for each experimental session. Note that as some participants might fall into multiple exclusion criteria, the totals may not add up exactly.

Table I Self-reported participant demographics and questionnaire-derived measures

\begin{tabular}{|c|c|c|c|c|c|c|}
\hline \multicolumn{3}{|l|}{ Measure } & All $(n=136)$ & COVID $(n=53)$ & Control $(n=83)$ & Statistic \\
\hline \multicolumn{3}{|c|}{ Age, mean years (SD) } & $28.6(9.7)$ & $28.0(8.6)$ & $29.0(10.3)$ & $t(I 34)=-0.6, P=0.5, B F=4.4$ \\
\hline \multicolumn{3}{|c|}{ Gender, female (\%) } & $54(39.7)$ & $23(43.4)$ & $31(37.3)$ & $X^{2}(I, N=54)=0.5, P=0.5$ \\
\hline \multirow[t]{5}{*}{ COVID-19 } & \multicolumn{2}{|c|}{$\begin{array}{l}\text { Time from COVID-19 diagnosis, } \\
\text { mean days (SD) }\end{array}$} & & $163.0(128.1)$ & & \\
\hline & \multirow{2}{*}{\multicolumn{2}{|c|}{$\begin{array}{l}\text { COVID-19 test type: } \\
\text { PCR/lateral flow/unknown/not } \\
\text { tested }\end{array}$}} & & $40 / 4 / 2 / 7$ & & \\
\hline & & & & & & \\
\hline & \multicolumn{2}{|c|}{$\begin{array}{l}\text { Stayed at hospital overnight for } \\
\text { COVID-19 treatment, yes (\%) }\end{array}$} & & $0(0.0)$ & & \\
\hline & \multicolumn{2}{|c|}{$\begin{array}{l}\text { Stayed at ICU for COVID-19 } \\
\text { treatment, yes (\%) }\end{array}$} & & $0(0.0)$ & & \\
\hline \multirow[t]{14}{*}{ Questionnaires } & \multirow[t]{4}{*}{ NFI } & Physical fatigue (SD) & $8.9(4.7)$ & $8.4(4.4)$ & $9.2(4.9)$ & $t(134)=-1.0, P=0.3, B F=3.4$ \\
\hline & & Cognitive fatigue (SD) & $4.2(2.8)$ & $4.0(2.8)$ & $4.4(2.8)$ & $t(I 34)=-0.7, P=0.5, B F=4 . I$ \\
\hline & & Sleep relief (SD) & $7.8(3.4)$ & $7.7(3.3)$ & $7.8(3.5)$ & $t(I 34)=-0.1, P=0.9, \mathrm{BF}=5.3$ \\
\hline & & Sleep abnormality (SD) & $6.9(2.8)$ & $7.2(2.9)$ & $6.7(2.7)$ & $t(134)=0.9, P=0.4, B F=3.8$ \\
\hline & \multirow[t]{3}{*}{ CFQ } & Forgetfulness (SD) & $12.7(5.0)$ & $13.0(4.8)$ & $12.4(5.2)$ & $t(86)=0.6, P=0.5, B F=4.4$ \\
\hline & & False triggering (SD) & $8.8(4.7)$ & $9.3(4.7)$ & $8.3(4.7)$ & $t(86)=1.0, P=0.3, B F=3.3$ \\
\hline & & Distractibility (SD) & $12.3(4.5)$ & $12.4(4.2)$ & $12.2(4.9)$ & $t(86)=0.1, P=0.9, B F=5.3$ \\
\hline & BFI-S & Conscientiousness (SD) & $10.5(2.5)$ & $10.4(2.3)$ & $10.7(2.7)$ & $t(77)=-0.6, P=0.6, B F=4.6$ \\
\hline & GRIT-S & Grit scale (SD) & $3.2(0.7)$ & $3.1(0.6)$ & $3.3(0.8)$ & $t(77)=-0.9, P=0.4, B F=3.7$ \\
\hline & \multirow[t]{3}{*}{ AMI } & Behavioural apathy (SD) & $10.3(4.4)$ & $10.9(4.5)$ & $9.8(4.2)$ & $t(77)=I . I, P=0.3, B F=3.0$ \\
\hline & & Social apathy (SD) & $12.9(5.0)$ & $12.5(5.4)$ & $13.4(4.4)$ & $t(77)=-0.8, P=0.4, B F=4 . I$ \\
\hline & & Emotional apathy (SD) & $8.0(4.2)$ & $8.6(4.4)$ & $7.3(3.9)$ & $t(77)=1.4, P=0.2, B F=2.2$ \\
\hline & \multirow[t]{2}{*}{ HADS } & Depression (SD) & $5.4(3.6)$ & $5.4(3.9)$ & $5.4(3.3)$ & $t(77)=0.0, P=1.0, B F=5.3$ \\
\hline & & Anxiety (SD) & $8.2(3.8)$ & $8.0(4.0)$ & $8.3(3.7)$ & $t(77)=-0.4, P=0.7, B F=4.9$ \\
\hline
\end{tabular}

$T$ - and $X^{2}$-tests were used to assess between-group differences, with Bayes Factor (BF) reported. The questionnaires included are Neurological Fatigue Index (NFI), Cognitive Failures Questionnaire (CFQ), Short Big Five Inventory (BFI-S), Short Grit Scale (GRIT-S), AMI and the Hospital Anxiety and Depression Scale (HADS). For all the questionnaire-derived indices, the mean score is shown with ISD in the bracket.

To compare the episodic memory deficit observed in the COVID group (see below), we ran the same memory test on healthy elderly people ( $>50$ years old). Sixty-four participants, who reported no neurological conditions, were recruited from the local community and have been involved in the lab's previous studies. All participants provided written informed consent and the study was approved by the University of Oxford ethics committee. Participants were 
initially contacted by emailed, followed up by calls and completed the task on their own computer devices. Fifty-three participants completed the task and one participant was excluded due to COVID-19 history. The results of 52 participants [30 females, mean age 67.4 years (SD 7.2)] are reported below.

\section{Questionnaires}

The questionnaires included were:

- Fatigue and sleep: Neurological Fatigue Index (NFI), which has been used to assess fatigue in persons with multiple sclerosis. ${ }^{18}$ Our motivation for including this index was driven by the fact that it probes the interactions between sleep and fatigue. Specifically, it asks questions related to abnormal nocturnal sleep, sleepiness and the need for diurnal sleep/rest.

- Distractibility and forgetfulness: Cognitive Failures Questionnaire (CFQ), ${ }^{19}$ a 25 -item questionnaire about minor mistakes in daily life over the last 2 weeks. For example: 'Do you find you forget appointments?' 'Do you fail to notice signposts on the road?' 'Do you find you forget which way to turn on a road you know well but rarely use?'.

- Personality: A Short 15-item Big Five Inventory (BFI-S) ${ }^{20}$ and the Short Grit Scale (GRIT-S). ${ }^{21}$ One of BFI-S components-conscientiousness-provides information about the conscientiousness personality trait, describing an individual's perseverance of effort combined with passions for a particular goal. To ensure that this is well-captured, we also included GRIT-S: this estimates the same trait but with different questions. We indeed found that these two scales were strongly positively correlated (Spearman's $\rho=0.65, P<0.0001$ ).

- Motivation: Apathy Motivation Index (AMI), an 18-item questionnaire, sub-divided into three subscales of apathy: emotional, behavioural and social apathy. ${ }^{22}$

- Mood: Hospital Anxiety and Depression Scale (HADS), a 14-item questionnaire, sub-divided into depression and anxiety. $^{23}$

All 155 participants completed the NFI questionnaire, of which 103 also completed the CFQ. 93 of these 103 participants completed the full set of questionnaires which were NFI, CFQ, BFI-S, GRIT-S, AMI and HADS.

\section{Vigilance test}

All participants were first tested on a version of an established, sustained visual attention task ${ }^{24}$ adapted into a modern online version hosted on the Pavlovia platform (pavlovia.org). An online demo is available at https://run. pavlovia.org/sijiazhao/vigilance_english_demo (open it with the Chrome Internet browser on a desktop computer). This task is designed to assess the performance decrement during sustained visual attention. A single digit (0-9) was presented at the centre of the screen for $50 \mathrm{~ms}$ every second (Fig. 2 Vigilance). Participants were instructed to press the spacebar on their keyboard as soon as they saw ' 0 ' (the target, presented randomly with a probability of $25 \%$ ); no response was required for other digits. A semi-transparent jittered checkerboard pattern masked the digits, with the level of difficulty manipulated by adjusting the opacity of the mask. Pilot testing allowed us to obtain an accuracy level of $\sim 80 \%$ in the first minute of the experiment. The practice phase consisted of 90 trials, equivalent to $90 \mathrm{~s}$. The difficulty was gradually increased and feedback was provided after each trial. The first 12 practice trials were highly visible stimuli and participants were required to get $100 \%$ accuracy to proceed. Subsequent non-practice trials and blocks contained no feedback. In total, each participant completed 540 trials, divided into nine blocks, each containing 60 trials and lasting $1 \mathrm{~min}$.

The experiment was implemented using PsychoPy v2021.1.2. ${ }^{25}$ To minimize the variance in latency caused by different browsers, all participants were required to use the Chrome browser on a desktop computer, although the operating system was not restricted. To minimize potential instability, the use of custom-written code was deliberately avoided and all functions used were as provided by PsychoPy. Only the questionnaires were implemented using in-house code.

\section{Motivation and fatigue ratings with time on the task}

After each minute during testing, participants were asked to report their level of fatigue ('How tired do you feel now?') and motivation ('How motivated do you feel?') using a visual analogue scale. Responses were registered by clicking on the appropriate position on each scale. After completing all ratings, a 'confirm' button appeared at the bottom of the screen, allowing participants to validate their ratings and start the next block. To control the time between blocks and to reduce variance between participants, a $15 \mathrm{~s}$ countdown timer was displayed at the top of the screen, and the next block would begin automatically once the timer lapsed. There were seven participants who had missing ratings. The exclusion of these participants did not affect the rating results or behavioural data and there were no group differences in age, gender or any questionnaire measures $(P>0.05)$.

\section{The session duration}

The session of the questionnaires and vigilance test took controls $22.4 \mathrm{~min}$ (SD 6.7) on average and COVID survivors $23.8 \mathrm{~min}$ (SD 6.4). There was no statistical difference in the session length between two groups $[t(135)=1.3, P=0.2]$. Although there was a relatively large variance in session duration amongst the participants, the duration of the main vigilance test was fixed at $11 \mathrm{~min} 15 \mathrm{~s}$ because each block was exactly 1 min plus a fixed break of $15 \mathrm{~s}$ (a countdown was shown on the screen). The participants could take breaks during the questionnaire and the practice, the length of which was not recorded. Importantly, the vigilance decrement (see below) did not correlate with the session duration (Spearman's $\rho=-0.06, P=0.5$ ). 

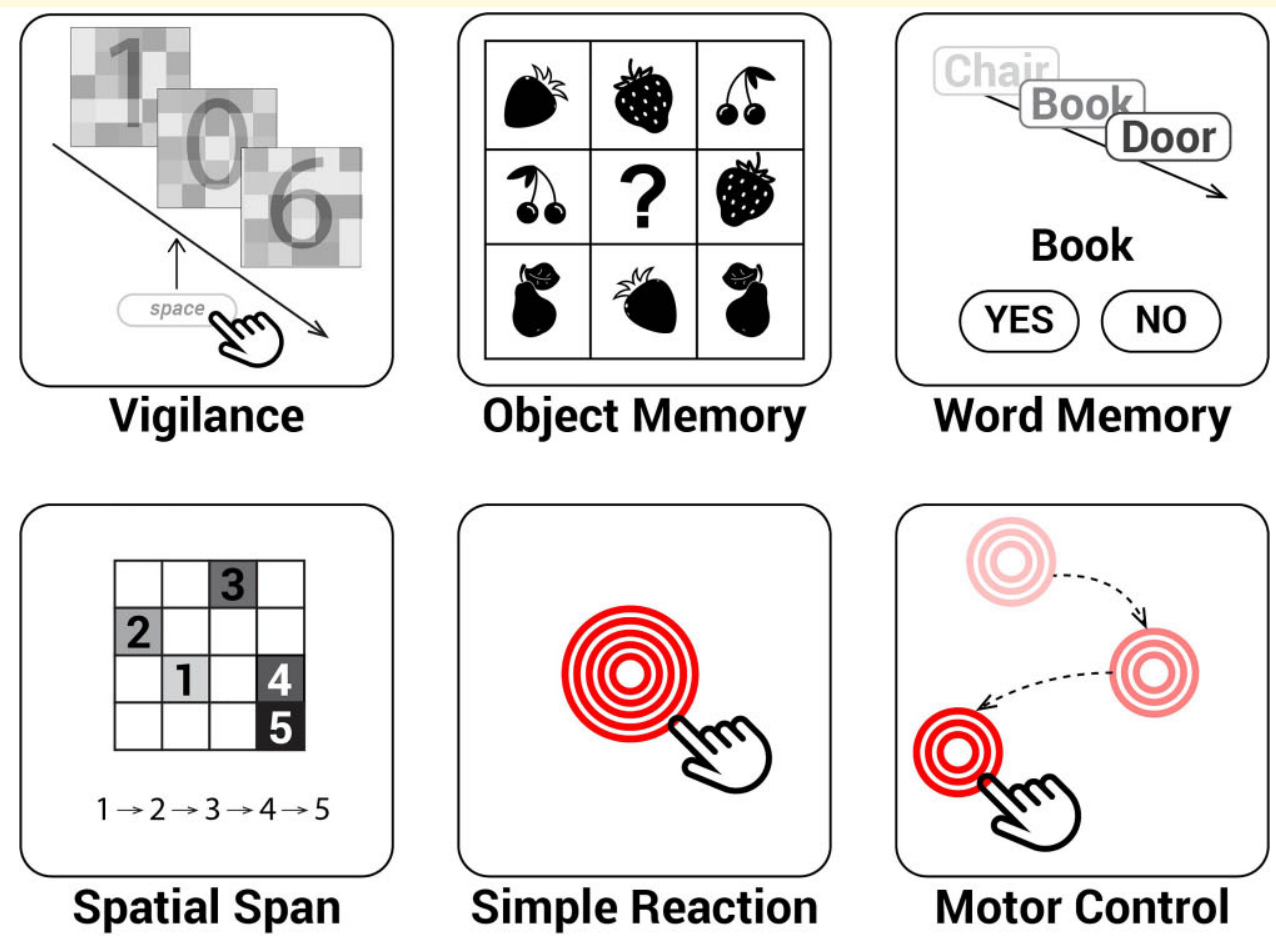
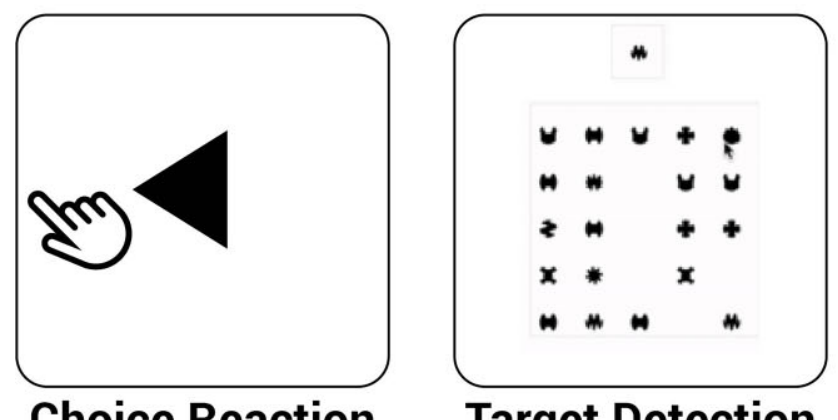

Target Detection

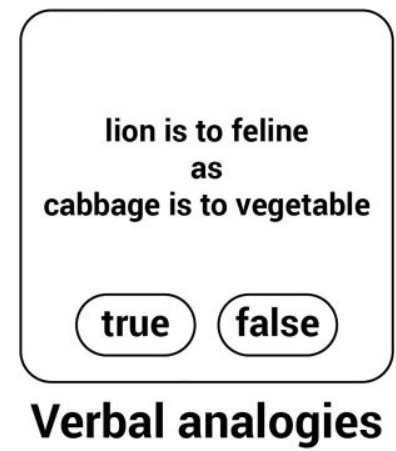

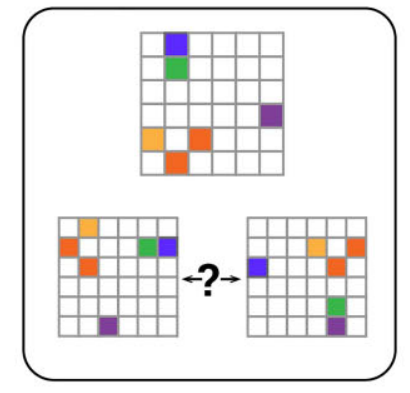

2D Mental Rotations

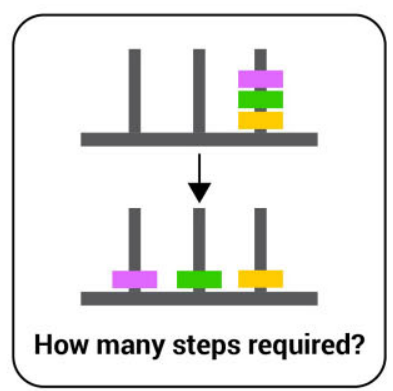

Tower of London

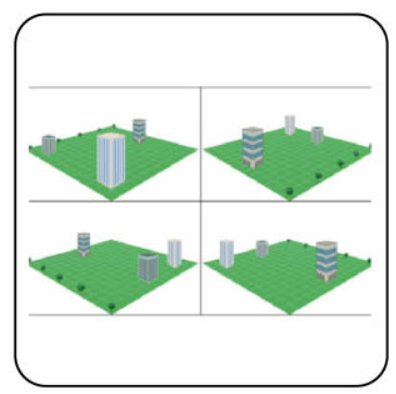

3D Mental Rotations

Figure 2 Twelve cognitive tasks measured distinct aspects of human cognition, memory, attention, motor control, planning and verbal reasoning abilities. The vigilance task was tested through the online experiment hosting server Pavlovia and conducted first. The rest of the II tasks were provided by Cognitron and ran in the following order: Motor control, Object Memory (immediate), Word memory (immediate), Simple reaction task, Choice reaction time, 2D mental rotations, 3D mental rotations, Spatial span, Target detection, Tower of London, Verbal analogies, Object Memory (delayed) and Word memory (delayed). The Object Memory and word memory tasks were both tested twice: once at the beginning ('immediate') and again at the end of the experiment ('delayed'), with an interval of about 30 min. The delayed task was solely testing memory of the stimuli displayed in the first instance of the task so the memory probes were not displayed before the delayed task. 


\section{Statistical analysis}

\section{Measuring of vigilance decrement}

The accuracy of each minute was measured as an F1 score, the harmonic mean of precision and sensitivity, given by

$$
F 1=\frac{2 \times \mathrm{TP}}{2 \times \mathrm{TP}+\mathrm{FP}+\mathrm{FN}}
$$

where TP is the true positive rate, FP is the false positive rate and $\mathrm{FN}$ is the false-negative rate. $F 1$ score is a particularly suitable accuracy measure for this task because it emphasizes true positives (responses to the rare target) and disregards true negatives (non-responses to non-targets). For reaction time (RT), only trials with correct responses were considered. In each block, trials with outliers (two standard deviations away from mean) were excluded, representing $3.7 \%$ of trials overall.

To investigate the effect of group and time on minute-wise performance and the three ratings, all the values were $z$-scored across participants and mixed-effects generalized linear models (GLM) were conducted using the MATLAB function fitglme with Laplace approximation as the method for estimating model parameters. The models included fixed effects of group and time and random effects of participants and their age.

The vigilance decrement for each individual was computed by subtracting the average accuracy over the first $3 \mathrm{~min}$ from the average over the last $3 \mathrm{~min}$ and then normalized by dividing the average over the first three blocks. The reason for using the average over the first three blocks as a performance baseline is because, in COVID-19 participants, the vigilance decrement happened relatively quickly, at the group level the accuracy was significantly lower than the first minute from the fourth minute (Fig. 3A).

\section{Time-series analysis}

To identify time intervals in which groups exhibit accuracy rate differences, a non-parametric bootstrap-based statistical analysis was used. ${ }^{26}$ In each iteration, $N$ data sets $(N=$ 53 here; based on the number of participants in the COVID group) were selected with replacement from each group and a difference between means was computed. These time series were subjected to bootstrap re-sampling (10000 iterations). At each time point, differences were deemed significant if the proportion of bootstrap iterations that fell above or below zero was more than $95 \%$ (i.e. $P<0.05)$. This analysis was applied to Fig. 3A-C.

\section{Group comparison}

$T$-test statistics and $\mathrm{BF}$ are reported. All $P$-values reported are two-tailed. When the sample sizes are not balanced, a bootstrap-based permutation test was used to confirm the reliability of the significance. For example, when comparing the vigilance decrement in participants who had COVID within 2 months $(N=13)$ versus the control participants $(N=73)$, in each iteration, $N$ data sets $(N=13$ here; based on the number of participants who had COVID-19 within 2 months) were selected with replacement from each group and a difference between means was computed. This process was then repeated 10000 times. Group difference was deemed significant if the proportion of bootstrap interactions that fell above or below zero was more than $95 \%$ (i.e. $P<0.05$ ).

\section{Correlation}

To control for outlier effects, all reported bivariate and partial correlations were performed using the conservative Spearman's rank correlation method. Additionally, we conducted partial correlations after controlling the effect of the grit scale of personality.

\section{Cognitive test battery}

On a subsequent occasion, a subset of participants also completed a sequence of 11 cognitive tasks to measure distinct aspects of human cognition, episodic memory, attention, motor control, planning, mental rotation and verbal reasoning abilities (Fig. 1). These 11 tasks can be viewed at https:// oxmh1.cognitron.co.uk. They were adapted from established behavioural paradigms in a manner that is sensitive to population variables of interest whilst being robust against the type of device that a person is tested on. The tasks were conceived, designed and programmed in HTML5 with JavaScript by A.H. and W.T., and hosted on a custom server system 'Cognitron' developed by P.J.H. on the Amazon EC2 platform.

This study was conducted independently to the vigilance task. All 155 participants were invited to attend this experiment and 81 completed this part of the study between 6 and 20 May 2021, with 36 from the COVID group [mean time since COVID-19 diagnosis: 233.8 days (129.1), age 27.4 (8.6), 14 females] and 44 from the control group [age 26.3 (8.0), 17 females]. No significant difference in age $[t(80)=$ $1.8, P=0.08, \mathrm{BF}=1.1]$ or gender $\left[\chi^{2}(1, N=31)=0.0005\right.$, $P=1.0]$ or any questionnaire-derived measures (all $P>$ 0.1 ) were observed in this subset of participants.

\section{Object episodic memory}

Participants were asked to memorize 20 images, all everyday objects, depicted in black and white. The images were presented sequentially in a random order, each for $2000 \mathrm{~ms}$ with an inter-image interval of $500 \mathrm{~ms}$. Immediately after the presentation, participants' memory of the 20 images was tested in 20 randomly ordered trials. Each trial required participants to select a previously displayed image from a set of eight images; incorrect images differed in the object itself, look or orientation (see the example in Fig. 2's Object Memory) in order to measure not only whether the correct target was identified, but also at higher precision the similarity of selected objects to the original target when errors were made. A delayed memory recall test was repeated at the end of the experiment, i.e. without additional sequential encoding of images; on average the time between the first and 

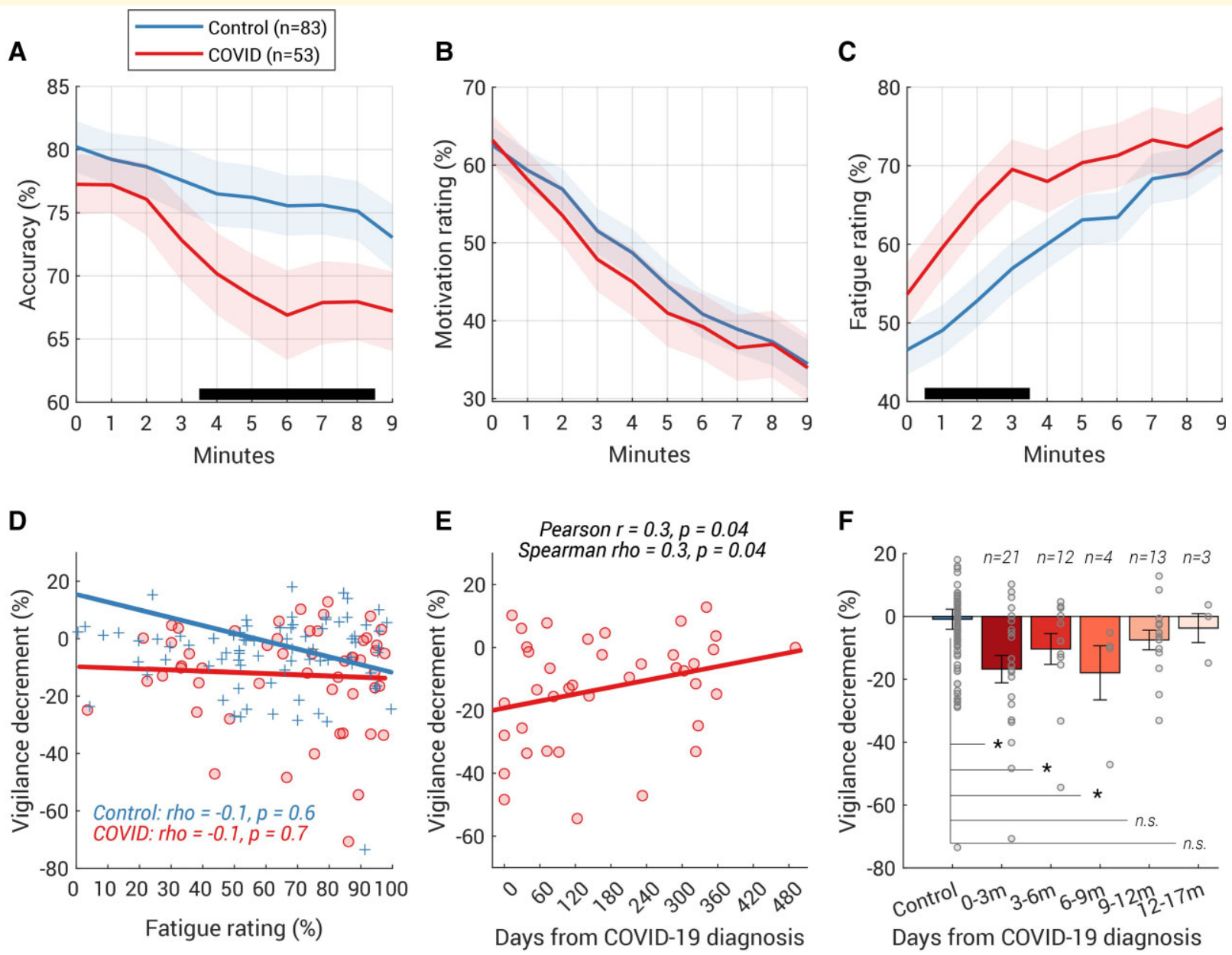

Figure 3 COVID group showed a larger and faster vigilance decline on the task. (A) Accuracy rate was computed for every minute (i.e. every block) of the vigilance test and plotted against the time. The $y$-value at $t=0$ corresponds to the accuracy rate over the I min-long practice block. The shaded area shows \pm I SEM and the black horizontal line at the bottom indicates time intervals where bootstrap statistics confirmed significant differences between the two groups $(P<0.05$, details see 'Time-series analysis' in 'Vigilance test'); the divergence was significant from the fourth minute to the eighth minute. (B) Group average of self-reported ratings of motivation against time (shaded area shows \pm I SEM). The rating at $t=0$ corresponds to the rating after the practice block. No group difference was found in the motivation rating over time. (C) COVID-I 9 survivors felt more tired from the beginning (shaded area shows \pm I SEM). (D) However, the fatigue rating (averaged over all I0 ratings) did not correlate with the size of the vigilance decrement in neither the COVID group nor the control group. The Spearman's correlation coefficient and their two-tailed $P$-values was shown for each group. (E) Vigilance decrement showed a significant correlation with the time from COVID-19 diagnosis. Both Spearman's and Pearson's correlation coefficients and their two-tailed $P$-values are shown above the plot. (F) Participants who had COVID-19 within the last 9 months displayed significantly larger vigilance decrements than the controls. The number of participants for each bin was labelled above each bar. Each grey dot represents individual data and the error bar indicates I SE. Group comparison performed by permutation test (with 10000 iterations). $* P<0.05$, m (months).

second test was $28.8 \mathrm{~min}$. Population mean of the immediate accuracy was $60.1 \%(20.9)$ compared with the delayed accuracy of $57.6 \%$ (21.0), both well above chance level $(12.5 \%)$. The memory decrement was computed by subtracting the accuracy in the immediate memory test from that in the delayed memory test for each individual.

\section{Word memory}

This task involved memorizing a sequence of English words (e.g. Peas, Monkey, Dress, Aubergine, Mouse, etc.). Words could be randomly drawn from three categories: animals, vegetables or clothes. The words were presented sequentially in a pseudo-random order, each presented for $1000 \mathrm{~ms}$ and with an inter-image interval of $200 \mathrm{~ms}$. Immediately postpresentation, word memory was tested with 24 words, including $50 \%$ non-targets, of which half were foils selected as being semantically similar to the targets [population mean of accuracy $90.9 \%(6.8)$ ]. After an average of $27.8 \mathrm{~min}$ later, participants were tested again to assess the episodic memory [mean accuracy $86.3 \%(9.1)]$. The memory decrement was computed by subtracting the accuracy in the immediate memory test from that in the delayed memory test for each individual. 


\section{Spatial span}

This task measured spatial short-term memory capacity. It was a variation of the Corsi Block Tapping Test ${ }^{27,28}$ participants were presented with a $4 \times 4$ grid, where each individual cell can light up sequentially. Participants were required to memorize the sequence and replicate it by clicking the appropriate squares in the order they lit up. The difficulty was incremented using a ratchet system, every time a sequence was recalled correctly, the length of the subsequent sequence was incremented by one. The test was terminated when three consecutive mistakes were made on a particular sequence length. The outcome measure was the maximum sequence length correctly recalled. Minimum level $=2$, maximum level $=16$, ISI $=0 \mathrm{~ms}$, encoding time $=1500 \mathrm{~ms}$. The mean sequence length successfully recalled was 6.6 (SD 1.3).

\section{Simple reaction task}

This task measured the basic cognitive process of perception and response execution. The participants were instructed to click on a red circle as soon as it appeared at the centre of the screen. They were presented with 60 stimuli, the ISI was jittered using a uniform random distribution between 0.5 and $2 \mathrm{~s}$. The dependent measure was the speed of response [population mean was $0.3 \mathrm{~s}$ (SD 0.1)].

\section{Motor control}

This task was identical to the reaction time task above, except that the location of the red circle was different each time. Participants had to move their cursor to the target and respond as fast as possible. The participants were presented with 30 stimuli, the next stimulus was presented with a 0 ms delay after the response to the previous stimulus. Here, the population mean was $0.7 \mathrm{~s}$ (SD 0.2).

\section{Choice reaction time}

A black arrow either pointing left or right appeared on-screen, indicating the side of the screen the participant needed to click as fast as possible. The participants were presented with 60 stimuli with a $50 \%$ chance of each stimulus pointing left. The ISI was jittered using a uniform random distribution between 0.5 and $2 \mathrm{~s}$. The mean RT over button presses for both sides was computed [population mean was $0.5 \mathrm{~s}(\mathrm{SD} 0.1)]$.

\section{Target detection}

This spatio-visual attention task involved identifying and clicking on a target shape amongst a field of distractor shapes. The participant was presented with a target shape on the left of the screen and a probe area on the right side of the screen. After $3 \mathrm{~s}$, the probe area began to fill with shapes, the participant must identify and click the target shape whilst ignoring the distractor shapes. Shapes were added every $1 \mathrm{~s}$ and a subset of the shapes in the probe area is removed every $1 \mathrm{~s}$. The trial ran for a total of 120 addition/removal cycles. The target shape was included in the added shapes pseudo-randomly, at a frequency of 12 in 20 cycles. The outcome measure was the total number of target shapes clicked. Population mean was 60.2 (SD 10.4).

\section{Tower of London}

This task measured spatial planning and was a variant of the original Tower of London task. ${ }^{29}$ The participant was shown two sets of three prongs with coloured beads on them. The first set was the initial state and the second set was the target state. The task was to work out the lowest number of moves it would take to transition from the initial state to the target state, then input this number using an on-screen number pad. The test consisted of 10 trials of variable difficulty, scaled using the number of beads and the convolutedness, defined as the number of moves that must be made that do not place a bead in its final target position. The outcome measure was the total number of correct trials. Unlike in the original task, ${ }^{29}$ the pegs were of equal height and the task was done mentally-the beads could not be moved. The total number of correct trials was recorded [population mean was 5.8 (SD 2.7)].

\section{Verbal analogies}

This task examined semantic reasoning. Participants were presented with two written relationships that they must decide if they had the same type of association or not (e.g. 'Lion is to feline as cabbage is to vegetable'). Participants indicated their decision by selecting the True or False buttons presented below the written analogies. Analogies were varied across semantic distance to modulate difficulty and association types switch throughout the sequence of trials. To obtain maximum points, participants must solve as many problems as possible within $3 \mathrm{~min}$. For every correct response, the total score increased by one. For every incorrect response, the total score decreased by one. The outcome measure was the total score [population mean total point score was 19.9 (SD 12.9)].

\section{Two-dimensional mental rotation}

The 2D mental rotation test measured the ability to spatially manipulate objects in the mind. ${ }^{30}$ In this version, we used $6 \times 6$ grids with various arrangements of coloured cells. Provided with a target grid and a set of four further grids, participants had to identify which of the four was the target but merely rotated by $90^{\circ}, 180^{\circ}$ or $270^{\circ}$ (incorrect grids had five incorrectly coloured cells). Participants were given $3 \mathrm{~min}$ to answer as many as possible, with correct trials being awarded with one point to their score [population mean was 34.5 (SD 8.5), technical issues beyond our control caused the loss of five participants' records for this task].

\section{Three-dimensional mental rotation}

Akin to the $2 \mathrm{D}$ version above, participants were tasked with recognizing a rotated version of the target from four options. In this 3D version, the grids were instead 3D scenes of buildings arranged upon a green surface. Again, the correct answer was identical to the target but from a different viewpoint (rotated by $90^{\circ}, 180^{\circ}$ or $270^{\circ}$ ), whilst the others 
had their buildings in the wrong locations. Each participant was presented with 12 trials scored in the same manner as before [population mean was 4.1 (SD 6.2)].

\section{The session duration}

This session took controls $37.4 \mathrm{~min}$ (SD 13.6) on average and COVID survivors $35.3 \mathrm{~min}$ (SD 11.7). There was no statistical difference in the session length between two groups $[t(94)=-0.8, P=0.4]$. The memory decrement (see below) did not correlate with the session duration (Spearman's $\rho=0.1, P=0.4)$.

\section{Statistical analysis}

For each cognitive function, a principal component analysis (PCA) was performed to derive a global index (PCA scores) quantifying its level across all relevant tasks used to assess it:

- Short-term memory score: PCA of full recall correct rate of Object Memory, full recall correct rate of Word Memory and Spatial Span memory capacity.

- Executive and attentional function score: PCA of reaction times in Simple Reaction Task, Motor Control and Choice Reaction Time and the overall score in Target Detection.

- Mental rotation score: PCA of scores in 2D and 3D mental rotation. This quantifies each individual's mental ability to spatially manipulate objects.

Group comparisons and correlations were performed as in Vigilance.

As the cognitive battery test was used as a broad-brush way of assessing a wide range of cognitive functions, Bonferroni correction was applied to the significant $P$-value(s). Unadjusted estimates would be provided if stated, along with adjusted estimates reported in the Results section.

\section{Ethics statement}

All participants gave electronic informed consent prior to the experiment. The study was approved by the local ethics committee and carried out in accordance with the relevant guidelines and regulations.

\section{Data availability}

The data supporting the findings of this study are available from the corresponding author upon reasonable request. A demo of the vigilance task is available at https://run. pavlovia.org/sijiazhao/vigilance_english_demo (Use the Chrome internet browser on a desktop computer). The experimental code is available at https://gitlab.pavlovia.org/ sijiazhao/vigilance_english_demo.

\section{Results}

COVID-19 survivors in this study were young, with many testing positive for COVID-19 several months before attending this study. Compared to the age-matched control group who did not report contracting COVID-19 before, there were no difference in gender or baseline group differences in a wide range of measures including fatigue/sleep abnormality (NFI), motivation (AMI), distractibility/ forgetfulness (CFQ), mood (HADS) and personality (GRIT-S and BFI-S) (Table 1).

Compared with the controls, COVID-19 survivors showed a significantly larger decline in performance in a 9 min-long sustained visual attention task (Fig. 1 Vigilance). There was no significant group difference in accuracy over the baseline [i.e. the first $3 \mathrm{~min}, t(135)=-0.9$, $P=0.4, \quad \mathrm{BF}=3.8]$. However, COVID-19 participants showed a greater and more rapid decline in accuracy over time [significant interaction between group and time using a mixed-effects $\operatorname{GLM}[F(1,1346)=18.8, P<0.0001]$ along with main effects of time $[F(1,1346)=15.1, P=0.0001]$ and group $[F(1,1346)=8.1, P=0.005)]$. Comparing the minute-by-minute accuracy rate (Fig. $3 \mathrm{~A}$ ), the group difference began to emerge by the start of the fourth minute and ended after the eighth minute.

On average, control participants' accuracy dropped from $78.5 \%$ (SD 20.2, average accuracy over the first $3 \mathrm{~min}$, see Table 2 for more details) to $75.4 \%$ (SD 20.9, average accuracy over the last $3 \mathrm{~min}$ ), whilst COVID survivors started with a similar baseline at $75.5 \%$ (19.2), reducing to $67.8 \%(23.0)$ by the end of the ninth minute. Comparing the absolute change in performance over time, COVID-19 survivors showed a significantly larger decline $[t(135)=-2.7, P=0.008, \mathrm{BF}=5.0]$. Importantly, we at tained a similar result after normalizing the change by individual's baseline performance [COVID $-12.3 \%$ (17.4) versus Control $-0.9 \%(29.1), t(135)=-2.6, P=0.01$, $\mathrm{BF}=3.8]$ suggesting that this larger vigilance decrement amongst the COVID group was regardless of the individual's baseline performance.

Across both groups, lower motivation (Fig. 3B) and greater fatigue (Fig. 3C) were reported as the experiment progressed (mixed-effects GLM, main effect of time $P<$ 0.001). Crucially, the COVID group showed a significantly faster fatigue accumulation [interaction of group $\times$ time, $F(1,1347)=8.8, \quad P=0.003]$ and significantly larger fatigue rating over time [main effect of group, $F(1,1347)=$ $5.7, P=0.02]$. Although the COVID group had a statistically similar fatigue rating before the experiment started $[t(131)=1.4, P=0.2, \mathrm{BF}=2.3$, COVID: $53.6 \%, \mathrm{SD} 29.5$, Control: $46.6 \%$, SD 28.7], they started to report a significantly higher fatigue rating after completing the first minute of the test (Fig. 3C), but these were not significant for motivation ratings (Fig. 3B). Noticeably, the fatigue ratingneither the average over all 10 ratings, the baseline rating, nor the change in the rating in the first minute-correlated with the vigilance decrement (Fig. 3D). This suggests that on an individual level, the vigilance decrement does not merely reflect the subjective feeling of being tired.

There was a significant positive correlation between individual vigilance decrement (normalized difference in average 
Table 2 Results of vigilance test

\begin{tabular}{|c|c|c|c|c|c|}
\hline \multicolumn{2}{|l|}{ Measures } & \multirow{2}{*}{$\begin{array}{c}\text { All } \\
(n=136) \\
77.3(19.8)\end{array}$} & \multirow{2}{*}{$\begin{array}{l}\text { COVID } \\
(n=53)\end{array}$} & \multirow{2}{*}{$\begin{array}{l}\text { Control } \\
(n=83)\end{array}$} & \multirow{2}{*}{$\begin{array}{c}\text { Statistic } \\
t\left(\begin{array}{l}(35)=-0.9, P=0.4, \mathrm{BF}= \\
3.8\end{array}\right.\end{array}$} \\
\hline Accuracy & Baseline accuracy (averaged over the first $3 \mathrm{~min}$ ), \% (SD) & & & & \\
\hline & Final accuracy (averaged over the first $3 \mathrm{~min}$ ), \% (SD) & $72.4(22.0)$ & $67.8(23.0)$ & $75.4(20.9)$ & $\begin{array}{l}t(I 35)=-2.0, P=0.05 \\
B F=I . I\end{array}$ \\
\hline & Accuracy over 9 min, \% (SD) & $74.7(20.0)$ & $71.4(19.8)$ & $76.8(20.0)$ & $\begin{array}{l}t(135)=-1.5, P=0.1, \mathrm{BF}= \\
\quad I .9\end{array}$ \\
\hline & Absolute change in accuracy (final minus baseline), \% (SD) & $-4.9(10.1)$ & $-7.7(10.3)$ & $-3.0(9.5)$ & $\begin{array}{l}t(135)=-2.7, P=0.008 \\
B F=5.0\end{array}$ \\
\hline & $\begin{array}{l}\text { Vigilance decrement, i.e. change in accuracy normalized by } \\
\text { individual baseline, mean \% (SD) }\end{array}$ & $-5.4(25.7)$ & $-12.3(17.4)$ & $-0.9(29.1)$ & $\begin{array}{l}t(I 35)=-2.6, P=0.0 I \\
\quad B F=3.8\end{array}$ \\
\hline \multirow[t]{3}{*}{ RT } & RT over 9 min, s (SD) & $0.5(0.1)$ & $0.5(0.1)$ & $0.5(0.1)$ & $\begin{array}{l}t(134)=-0.2, P=0.8, B F= \\
5.2\end{array}$ \\
\hline & Absolute change in RT (final minus baseline), s (SD) & $0.02(0.1)$ & $0.01(0.1)$ & $0.02(0.1)$ & $\begin{array}{l}t(130)=-0.8, P=0.4, B F= \\
\quad 4.0\end{array}$ \\
\hline & Change in RT normalized by individual baseline, \% (SD) & $4.0(10.3)$ & $3.0(12.2)$ & $4.6(8.9)$ & $\begin{array}{l}t(I 30)=-0.9, P=0.4, B F= \\
\quad 3.8\end{array}$ \\
\hline \multirow[t]{6}{*}{ Ratings } & Baseline fatigue rating (average over the first $3 \mathrm{~min}$ ), \% (SD) & $57.7(28.6)$ & $65.1(27.2)$ & $52.8(28.6)$ & $\begin{array}{l}t(I 35)=2.5, P=0.0 I, B F \\
\quad=3 . I\end{array}$ \\
\hline & Fatigue rating, average over $9 \min \%(S D)$ & $63.3(26.2)$ & $68.2(25.7)$ & $60.1(26.1)$ & $\begin{array}{l}t(135)=1.8, \quad P=0.08, \quad B F \\
\quad=1.3\end{array}$ \\
\hline & Change in fatigue rating (last 3 min minus first $3 \mathrm{~min}$ ), \% (SD) & $11.6(17.5)$ & $7.9(17.4)$ & I4.I (17.2) & $\begin{array}{l}t(135)=-2.1, P=0.04, \mathrm{BF} \\
\quad=1.3\end{array}$ \\
\hline & Baseline motivation rating, \% (SD) & $54.6(24.6)$ & $52.9(26.4)$ & $55.7(23.4)$ & $\begin{array}{l}t(135)=-0.7, P=0.5, B F= \\
\quad 4.4\end{array}$ \\
\hline & Motivation rating, average over all ratings\% (SD) & $46.3(24.5)$ & $45.2(26.4)$ & $47.0(23.3)$ & $\begin{array}{l}t(135)=-0.4, P=0.7, \mathrm{BF}= \\
\quad 4.9\end{array}$ \\
\hline & Change in motivation rating (last $3 \mathrm{~min}$ minus first $3 \mathrm{~min}$ ), \% (SD) & $-16.3(17.8)$ & $-15.7(17.0)$ & $-16.7(18.4)$ & $\begin{array}{l}t(135)=0.3, \quad P=0.8, \quad B F \\
\quad=5.1\end{array}$ \\
\hline
\end{tabular}

$T$-tests used to assess between-group differences, with Bayes Factor (BF) reported. The significant $t$-tests are highlighted in bold.

accuracy between first and last $3 \mathrm{~min}$ ) and time from COVID diagnosis (Fig. 3E, $\rho=0.3, P=0.04$, two-tailed; partial correlation after controlling age and personality of grit scale: $\rho=0.4, P=0.03$, two-tailed). Furthermore, participants who had COVID-19 in the last 9 months (Fig. 3F) showed a significantly larger vigilance decrement than the control group $[n=37, t(118)=-2.7, P=0.009, \mathrm{BF}=4.7$, bootstrap-based $P=0.001$ ], with this difference no longer apparent in participants who had COVID-19 more than 9 months previously $[n=16, t(97)=-0.8, P=0.4, \mathrm{BF}=2.7$, bootstrap-based $P=0.2$ ].

In order to obtain a more comprehensive examination of our COVID-19 survivors' cognitive profiles, we subsequently invited all participants to complete a cognitive battery comprising 11 cognitive tests (all bar Vigilance in Fig. 2) 2 months after the Vigilance test. Thirty-six COVID-19 survivors had been diagnosed on average 233.8 days previously (129.1) and 44 age-matched controls attended the battery (Table 3 for demographics).

Memory was a key cognitive function measured in this battery. In one of the memory tests-Object Memoryparticipants were shown 20 images of everyday objects to memorize. Specifically, participants needed to remember not only the object (e.g. a spoon), but also its look (e.g. a spoon with a long handle) as well as its orientation (e.g. handle pointing towards top-right). This provides us details about the preciseness of the memory recalled. One novel feature of this test is that Object Memory was tested twice, once immediately after presentation and again around $30 \mathrm{~min} \mathrm{la-}$ ter, which provides a measure of memory decrement over time.

On average, the controls achieved $59.7 \%$ accuracy in the immediate memory test. COVID-19 survivors showed a similar short-term memory performance to the control group $[t(78)=-0.02, P=1.0, \mathrm{BF}=4.3$; Fig. $4 \mathrm{~A}$ and see Table 3 for more details]. However, a difference emerged in the later (delayed) test; whilst the controls displayed no memory decrement [no difference from zero, one sample $t$-test: $t(43)=$ $0.9, P=0.4, \mathrm{BF}=5.8]$, COVID-19 survivors showed a significant memory decrement $[t(35)=-4.1, P=0.0003$, $\mathrm{BF}=107.5]$, which was larger than in controls by $9.2 \%$ $[t(78)=3.3, P=0.001, \mathrm{BF}=23.2$; Fig. $4 \mathrm{~B}]$. This group difference would survive through Bonferroni correction (29 statistical tests were done for all 11 cognitive battery tests, see Tables 3 and 4 , adjusted $P=0.029$ ). Like the vigilance decrement, the memory decrement is computed as a normalized change in performance (i.e. the difference between delayed and immediate memory test, divided by the immediate memory test). Importantly, this difference in episodic memory decrement was not due to the variance in memory maintenance duration, because there was no difference in the duration between the immediate and delayed 
Table 3 Demographics and the results of the Object Memory task in the battery test

\begin{tabular}{|c|c|c|c|c|c|c|}
\hline \multicolumn{3}{|l|}{ Measure } & $\begin{array}{c}\text { All } \\
(n=80)\end{array}$ & $\begin{array}{l}\text { COVID } \\
(n=36)\end{array}$ & $\begin{array}{l}\text { Control } \\
(n=44)\end{array}$ & Statistic \\
\hline \multicolumn{3}{|c|}{ Age, years, mean (SD) } & $26.8(8.2)$ & $27.4(8.6)$ & $26.3(8.0)$ & $\begin{aligned} t(78) & =0.6, P=0.6 \\
B F & =3.7\end{aligned}$ \\
\hline \multicolumn{3}{|c|}{ Gender, female, $n(\%)$} & $31(38.8)$ & $14(38.9)$ & $17(38.6)$ & $\begin{array}{l}X^{2}(I, N=3 \mathrm{I})=0.0 \\
\quad P=I .0\end{array}$ \\
\hline \multirow[t]{4}{*}{ COVID-19 } & \multicolumn{2}{|c|}{ Time from COVID-19 diagnosis, mean days (SD) } & & $233.8(129.1)$ & & \\
\hline & \multicolumn{2}{|c|}{ COVID-19 test type: PCR/lateral flow/unknown/not tested } & & \multicolumn{2}{|l|}{$26 / 2 / 3 / 5$} & \\
\hline & \multicolumn{2}{|c|}{ Stayed at hospital overnight for COVID-I 9 treatment, yes (\%) } & & $0(0.0)$ & & \\
\hline & \multicolumn{2}{|c|}{ Stayed at ICU for COVID-I9 treatment, yes (\%) } & & $0(0.0)$ & & \\
\hline \multirow[t]{6}{*}{$\begin{array}{l}\text { Object } \\
\text { Memory }\end{array}$} & \multirow[t]{2}{*}{ Immediate memory test } & Accuracy, mean \% (SD) & $60.1(20.9)$ & $60.0(15.9)$ & $60.1(24.4)$ & $\begin{aligned} t(78) & =-0.02, P=1.0, \\
B F & =4.3\end{aligned}$ \\
\hline & & RT in $s$, mean (SD) & $3.3(1.8)$ & $3.2(1.6)$ & $3.4(2.0)$ & $\begin{aligned} t(78) & =-0.4, P=0.7 \\
B F & =4.0\end{aligned}$ \\
\hline & \multirow[t]{2}{*}{ Delayed memory test } & Accuracy, mean \% (SD) & $57.6(21.0)$ & $52.5(20.6)$ & $61.8(20.7)$ & $\begin{array}{c}t(78)=-2.0, P= \\
0.05, B F=I .3\end{array}$ \\
\hline & & $\mathrm{RT}$ in $\mathrm{s}$, mean (SD) & $2.3(0.8)$ & $2.2(0.6)$ & $2.4(1.0)$ & $\begin{aligned} t(78) & =-1.3, P=0.2 \\
B F & =2.1\end{aligned}$ \\
\hline & $\begin{array}{l}\text { Memory decrement (normalized } \\
\text { by immediate accuracy) }\end{array}$ & $\begin{array}{l}\text { Percentage change in } \\
\text { accuracy, mean \% (SD) }\end{array}$ & $3.0(46.8)$ & $-13.6(21.4)$ & I6.6 (56.9) & $\begin{array}{c}t(78)=-3.0, P= \\
0.003, B F=10.7\end{array}$ \\
\hline & $\begin{array}{l}\text { Time between immediate and } \\
\text { delayed tests }\end{array}$ & $\begin{array}{l}\text { Duration in minutes, mean } \\
\text { (SD) }\end{array}$ & $28.8(9.4)$ & 27.9 (8.7) & $29.6(9.9)$ & $\begin{aligned} t(78) & =-0.8, P=0.4 \\
B F & =3.2\end{aligned}$ \\
\hline
\end{tabular}

The time from COVID-19 diagnosis was computed as the days between the date they attended this battery test and the self-reported date of their positive COVID-19 test. $T$ - and $\mathrm{X}^{2}$ tests used to assess between-group differences, with Bayes Factor (BF) reported. The significant $t$-tests are highlighted in bold.

memory tests across the two groups [COVID: $27.9 \mathrm{~min}(8.7)$, control: $29.6 \min (9.9), t(78)=-0.8, P=0.4, \mathrm{BF}=3.2]$.

Furthermore, the larger episodic memory decrement amongst COVID-19 survivors was driven by errors in which the wrong orientation was chosen for a correct item. In the immediate memory test, COVID-19 survivors had a $30.6 \%$ (13.3) false alarm rate where they chose the right object but wrong orientation, misbinding object identity with object orientation. This was not significantly different from controls [28.6 (18.3), $t(80)=0.5, P=0.6, \mathrm{BF}=3.8]$. However, this orientation-specific false alarm rate increased to $35.3 \%$ (15.7) 30 min later in the COVID group, which was significantly higher than the controls $[27.3 \%(14.7), t(80)=2.4$, $P=0.02, \mathrm{BF}=2.6]$. This difference suggests that the deficit in episodic memory in the COVID group might be associated with a deficit in binding information in memory.

In another memory test (Fig. 2 Word Memory), participants were instructed to memorize 24 simple English words. Although both COVID and control groups showed significant memory decrement [COVID: $t(35)=-4.5, P=$ 0.00008, $\mathrm{BF}=305.0$; control: $t(43)=-4.4, P=0.00007$, $\mathrm{BF}=314.5]$, they did not differ significantly from each other $[t(78)=-0.04, P=1.0, \mathrm{BF}=4.3]$. Because our participants reported having different first languages, we also ran a 2 (group: COVID versus control) $\times 2$ (first language: English or non-English) ANOVA on the memory decrement. There were no main effects of first language $[F(1,76)=1.1, P=$ $0.3]$ or group $[F(1,76)=0.1, P=0.7]$ and no languagegroup interaction $[F(1,76)=0.1, P=0.7]$, indicating that this null effect was not due to differences in first language amongst participants. This null effect in word memory decrement might be due to the fact that the word memory task was much simpler than Object Memory which also had precision manipulation.

As part of a separate study, we ran the same memory tests on 52 healthy elderly participants [Elderly Control group: 53-82 years old, mean 67.4 (7.2), 30 females, no self-report COVID-19]. Amongst controls $(N=96$, including all from Control and Elderly Control groups), there was a weak correlation between memory decrement and age (Pearson $r=-0.2, P=0.03$; Spearman's $\rho=-0.2, P=$ 0.07). Compared with young controls, elderly participants had a significantly larger memory decrement [mean (SD): -7.1 (21.8), $t(94)=2.8, P=0.007, \mathrm{BF}=6.0$, Fig. $4 \mathrm{~B}]$, but importantly elderly participants' episodic memory decrement was not statistically different from COVID-19 survivors $[t(86)=-1.4, P=0.2, \mathrm{BF}=1.9$, Fig. 4B], indicating that COVID-19 survivors performed as if they were older.

However, the elderly controls spent a longer time completing other tasks between the immediate and delayed memory tests [mean 39.2 (6.1) minutes] than the COVID group $\left[t(86)=-7.2, P<10^{9}, \mathrm{BF}>10^{7}\right]$. The requirement to maintain memory for a longer period was associated with greater memory decrements (partial correlation of time between memory tests and memory decrement amongst young and elderly controls after controlling the effect of age: Pearson $r=0.2, P=0.03$; Spearman's $\rho=0.05, P=0.6)$. Therefore, we regressed out the effect of the memory maintenance time from each individual's memory decrement, but the pattern remained unchanged.

Amongst survivors who contracted COVID-19 within the last year, the size of memory decrement was weakly but significantly correlated with the time from diagnosis (Fig. 4C, Pearson $r=0.6, P=0.001$; Spearman $\rho=0.5, P=0.008$ ), 

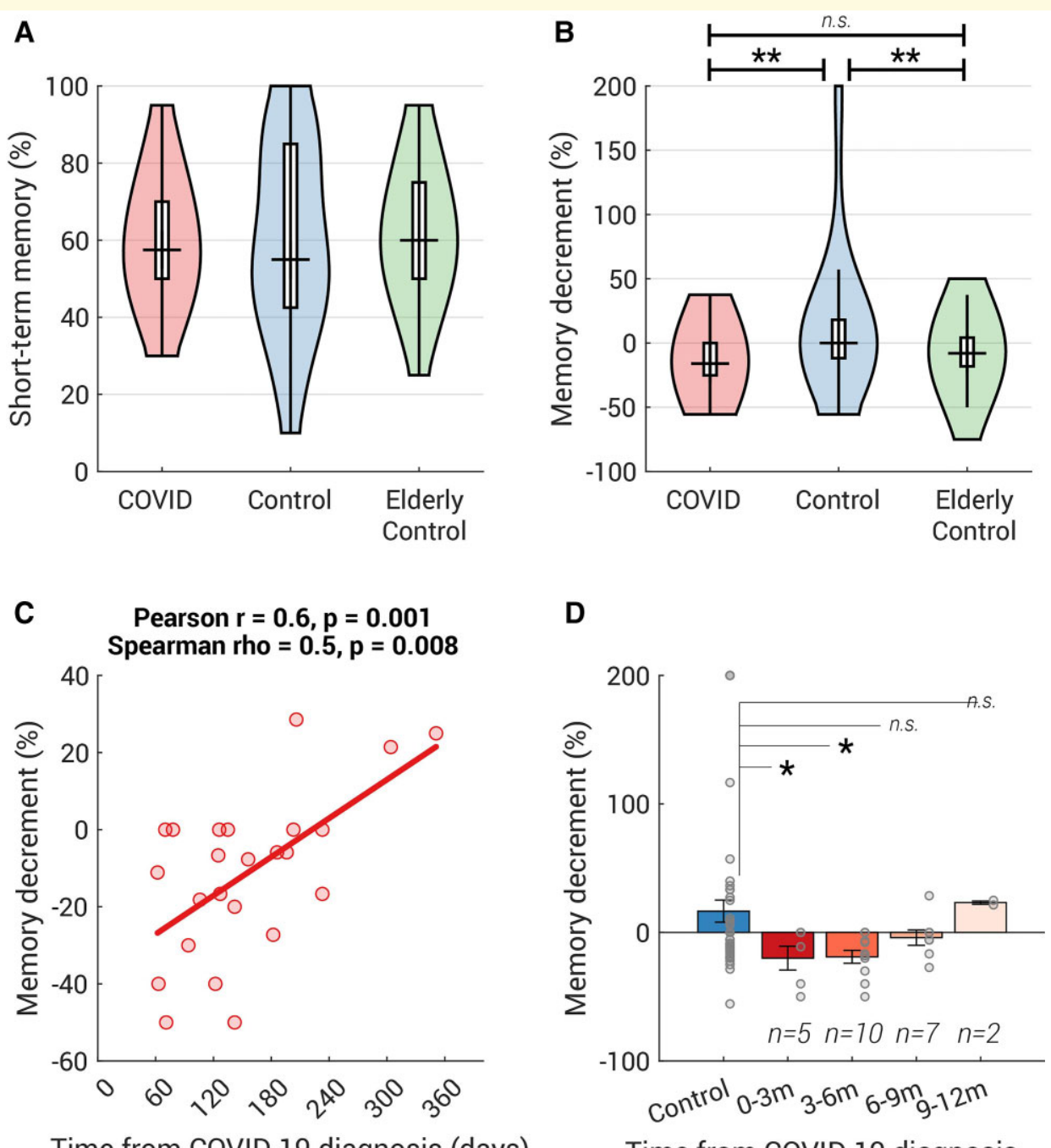

Time from COVID-19 diagnosis (days)

\section{Time from COVID-19 diagnosis}

Figure 4 COVID group showed a mild episodic memory deficit compared with age-matched controls. (A) The distribution of the short-term memory, measured as the correct percent in the memory test immediately after viewing the sequence of objects, is plotted as a violin for COVID $(n=36)$, Control $(n=44)$ and Elderly Control $(n=52$, all above 50 years old, data collected separately). Group comparison performed by $t$-test. There were no statistical differences between groups in the short-term memory [COVID versus Control: $t(78)=-0.02, P=$ I.0, $\mathrm{BF}=4.3$; Control versus Elderly Control: $t(94)=-0.4, P=0.7, \mathrm{BF}=4.4$; COVID versus Elderly Control: $t(86)=-0.5, P=0.6, \mathrm{BF}=4.0]$. (B) Approximately $30 \mathrm{~min}$ later, their memory was tested again. COVID and Elderly controls showed significantly larger memory decrements than the younger controls [COVID versus Control: $t(78)=-3.0, P=0.004, \mathrm{BF}=10.7$; Control versus Elderly Control: $t(94)=2.8, P=0.007, \mathrm{BF}$ $=6.0$; COVID versus Elderly Control: $t(86)=-1.4, P=0.2, B F=1.9]$. (C) In COVID-19 survivors who contracted COVID-I9 within I year, the size of memory decrement was positively correlated with the time from COVID-19 diagnosis. Both Spearman's and Pearson's correlation coefficients and their two-tailed P-values are shown above the plot. (D) Participants who had COVID-19 within the last 6 months showed significantly larger memory decrement than the age-matched controls. The number of participants for each bin was labelled above each bar. Each grey dot represents individual data and the error bar indicates I SE. Group comparison performed by permutation test (with 10000 iterations). $* P<0.05, * * P<0.01, m$ (months).

suggesting that people who had COVID-19 more recently tended to forget more over the 30-min interval. This significant memory decrement could be observed up to 6 months $[N=15, t$-test: $t(57)=-2.4, P=0.02$; Fig. 4D]. These analyses provide preliminary evidence that COVID-associated reductions in sustained attention and episodic memory may persist for months, but may normalize subsequently, although the findings have to be taken with caution given the sample size.
Do these cognitive differences relate to the symptom severity experienced during COVID-19 illness or the postillness long-COVID? In a follow-up survey, we asked the participants for their experience during and after their COVID-19 illness (Fig. 5A and C). The questions were modified from Office for National Statistics-Coronavirus (COVID-19) Infection Survey; for example, the question about the COVID-19 symptom severity was 'Do any of the COVID-19 symptoms reduce your ability to carry out 
Table 4 Results of the 10 cognitive tasks in the battery test

\begin{tabular}{|c|c|c|c|c|c|c|}
\hline Measure & & & $\begin{array}{c}\text { All } \\
(n=80)\end{array}$ & $\begin{array}{l}\text { COVID } \\
(n=36)\end{array}$ & $\begin{array}{l}\text { Control } \\
(n=44)\end{array}$ & Statistic \\
\hline \multirow[t]{6}{*}{ Word memory } & Immediate memory test & Accuracy, mean \% (SD) & $90.9(6.8)$ & 91.1 (7.1) & $90.7(6.7)$ & $\begin{aligned} t(78) & =0.2, P=0.8, \\
B F & =4.2\end{aligned}$ \\
\hline & & RT in $s$, mean (SD) & $1.0(0.3)$ & $1.0(0.3)$ & $1.0(0.2)$ & $\begin{aligned} t(78) & =0.2, P=0.8, \\
B F & =4.2\end{aligned}$ \\
\hline & Delayed memory test & Accuracy, mean \% (SD) & $86.3(9.1)$ & $86.3(8.6)$ & $86.2(9.5)$ & $\begin{aligned} t(78) & =0.1, P=0.9, \\
B F & =4.3\end{aligned}$ \\
\hline & & $\mathrm{RT}$ in $\mathrm{s}$, mean (SD) & $0.9(0.2)$ & $0.8(0.2)$ & $0.9(0.2)$ & $\begin{array}{l}t(78)=-I . I \\
\quad P=0.3, B F=2.6\end{array}$ \\
\hline & $\begin{array}{l}\text { Memory decrement (normalized } \\
\text { by immediate accuracy) }\end{array}$ & $\begin{array}{l}\text { Percentage change in } \\
\text { accuracy, mean \% (SD) }\end{array}$ & $-5.1(46.8)$ & $-5.1(21.4)$ & $-5.1(56.9)$ & $\begin{array}{l}t(78)=-0.04 \\
\quad P=1.0, B F=4.3\end{array}$ \\
\hline & $\begin{array}{l}\text { Time between immediate and } \\
\text { delayed tests }\end{array}$ & $\begin{array}{l}\text { Duration in minutes, mean } \\
\text { (SD) }\end{array}$ & $27.8(8.4)$ & $26.7(6.8)$ & $28.8(9.5)$ & $\begin{array}{l}t(78)=-I . I \\
\quad P=0.3, B F=2.6\end{array}$ \\
\hline $\begin{array}{l}\text { Spatial short-term } \\
\text { memory capacity }\end{array}$ & Spatial span & $n$, mean $(S D)$ & $6.6(1.3)$ & $6.4(1.4)$ & $6.8(1.3)$ & $\begin{array}{l}t(78)=-1.2 \\
\quad P=0.2, B F=2.3\end{array}$ \\
\hline \multirow[t]{3}{*}{ Motor control } & Simple reaction time & $\mathrm{RT}$ in $\mathrm{s}$, mean (SD) & $0.3(0.1)$ & $0.3(0.1)$ & $0.3(0.1)$ & $\begin{array}{l}t(78)=-1.2 \\
\quad P=0.2, B F=2.2\end{array}$ \\
\hline & Motor control & $\mathrm{RT}$ in $\mathrm{s}$, mean (SD) & $0.7(0.2)$ & $0.7(0.2)$ & $0.7(0.2)$ & $\begin{aligned} t(78) & =0.5, P=0.6, \\
B F & =3.9\end{aligned}$ \\
\hline & Choice reaction time & $\mathrm{RT}$ in $\mathrm{s}$, mean (SD) & $0.5(0.1)$ & $0.5(0.1)$ & $0.5(0.1)$ & $\begin{array}{l}t(78)=-0.2 \\
\quad P=0.9, B F=4.2\end{array}$ \\
\hline $\begin{array}{l}\text { Spatial-visual } \\
\text { attention }\end{array}$ & Target detection & Score, mean (SD) & $60.2(10.4)$ & $60.4(11.0)$ & $60.1(10.0)$ & $\begin{aligned} t(78) & =0.1, P=0.9, \\
B F & =4.3\end{aligned}$ \\
\hline Spatial planning & Tower of London & Score, mean (SD) & $5.8(2.7)$ & $5.8(2.7)$ & $5.9(2.7)$ & $\begin{array}{l}t(78)=-0.2 \\
\quad P=0.8, B F=4.2\end{array}$ \\
\hline Semantic reasoning & Verbal analogies & Score, mean (SD) & $19.9(12.9)$ & $18.3(1 \mid .2)$ & $21.2(14.2)$ & $\begin{array}{l}t(78)=-1.0 \\
\quad P=0.3, B F=2.8\end{array}$ \\
\hline \multirow[t]{2}{*}{ Mental rotation } & 2D mental rotation & Score, mean (SD) & $34.5(8.5)$ & $34.7(7.4)$ & $34.4(9.3)$ & $\begin{aligned} t(73) & =0.2, P=0.9, \\
B F & =4.2\end{aligned}$ \\
\hline & $3 \mathrm{D}$ mental rotation & Score, mean (SD) & $4.1(6.2)$ & $4.3(6.1)$ & $4.0(6.3)$ & $\begin{aligned} t(78) & =0.3, P=0.8, \\
B F & =4.2\end{aligned}$ \\
\hline
\end{tabular}

Results from the same 80 participants reported in Table 3 are shown. $T$ - and $X^{2}$-tests used to assess between-group differences, with Bayes Factor (BF) reported. Note that due to the technical issue, we lost five participants' data for the 2D mental rotation task, thus resulting in a smaller degree of freedom in the $t$-test for that task.

day-to-day activities?' with four options: Yes, a lot/Yes, a little/Not at all/No symptom, which in turn corresponds to Severe/Moderate/Mild/Asymptomatic. To assess the relation between the post-COVID cognitive decrements observed here and the COVID-19/long-COVID severity, a linear mixed-effect model (LMM) with COVID-19 severity level and long-COVID severity level as fixed effects, and participant as a random effect was applied. Amongst the COVID-19 survivors who had any COVID-19 or long-COVID symptoms, larger vigilance and memory decrements were associated with more severe COVID-19 symptoms [Fig. 5B, main effect on vigilance decrement: $F(1,8)=$ 6.8, $P=0.03$; main effect on memory decrement: $F(1,6)=$ 15.3, $P=0.008$ ] but not with long-COVID symptoms [Fig. 5D, main effect on vigilance decrement: $F(1,8)=4.5$, $P=0.07$; main effect on memory decrement: $F(1,6)=0.09$, $P=0.8]$. However, two caveats require attention: first, the positive relation between the COVID-19 symptom severity and the cognitive decrements must be taken with caution because it disappears if taking asymptotic participants into account. Secondly, the null effect of long-COVID severity on the cognitive decrements might be specific to the present study as most of COVID-19 survivors in the present study did not have any long-COVID symptoms. Nevertheless, further confirmation of these relations is out of scope of the present study and should be addressed in patient studies amongst COVID-19 inpatients and/or long-COVID patients.

Although the correlation between cognitive decrement and time since COVID-19 diagnosis (Figs 3E and 4D) provides strong evidence linking cognitive differences observed in the present with COVID-19 infection, we additionally considered additional non-infectious factors. First, we ruled out basic factors including age [Fig. 6A, $t(134)=$ $-0.6, P=0.5$ ], gender [Fig. $6 \mathrm{~B}, \chi^{2}(1, N=54)=0.5, P=$ 0.5 ], first language [Fig. $6 \mathrm{C}, \chi^{2}(1, N=50)=0.03, P=0.9$ ], country of current residence [Fig. $6 \mathrm{D}, \chi^{2}(1, N=36)=0.2$, $P=0.7$ ] and ethnicity (Fig. 6E, all categories $P>0.1$ ), as none showed any difference between the two groups. Secondly, we pondered whether the more significant decline in attention and memory of the COVID group could be attributed to a lower socioeconomic status (SES). This is highly possible: a recent study ${ }^{31}$ suggested strong associations between low SES and high probability of COVID-19 infection, along with higher infection fatality rate. Meanwhile, lower SES has established negative pressures on cognition including attention and memory $\left(\right.$ see review $\left.{ }^{32}\right)$. To address this concern, all participants received a follow-up survey covering a wide range of demographic and 
A COVID-19 symptom severity

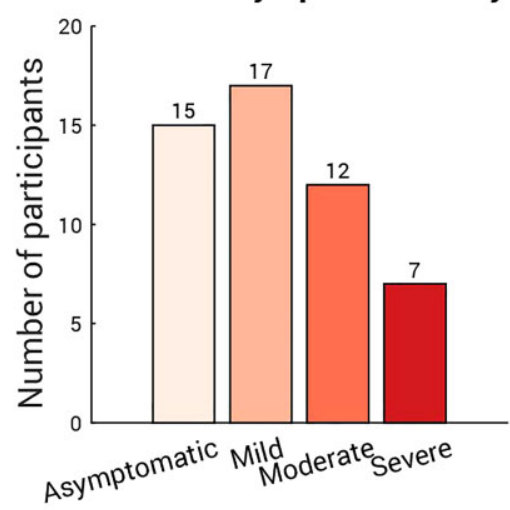

C Long-COVID symptom severity

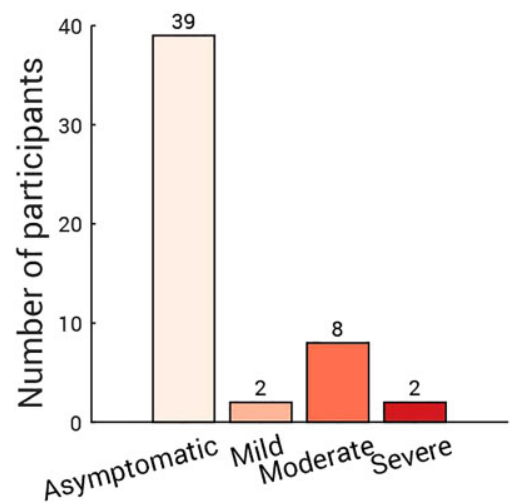

B COVID-19 symptom severity

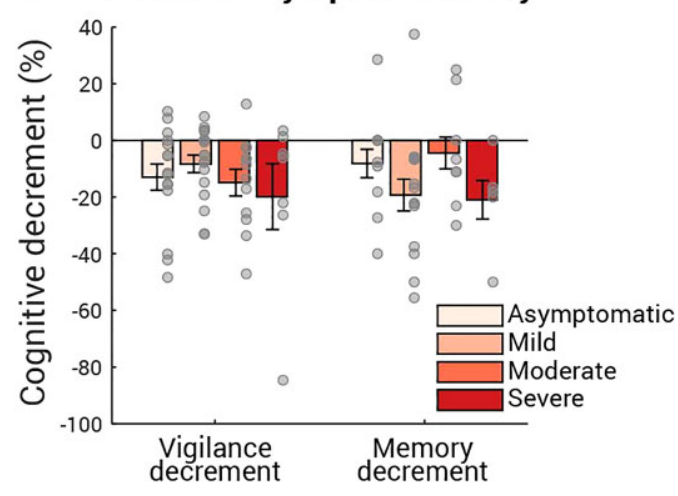

D Long-COVID symptom severity

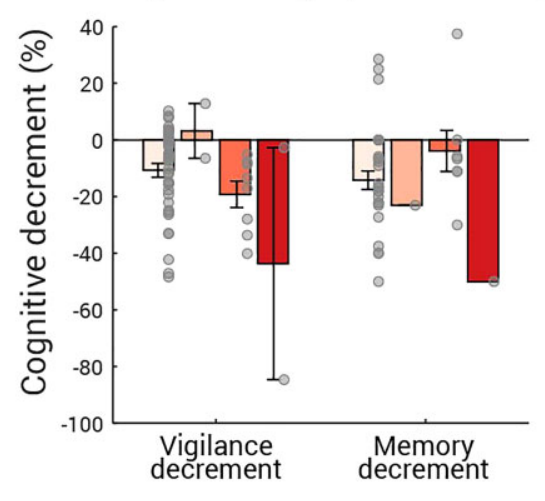

Figure 5 Cognitive decrements sorted by COVID- 19 symptom and long-COVID symptom severity. Fifty-one out of 64 COVID-19 survivors (including the three participants who stayed hospital overnight for COVID-19) reported their COVID-19 symptom severity (A) and long-COVID symptom severity $(\mathbf{C})$. In both $(\mathbf{A})$ and $(\mathbf{C})$, the number of participants for each severity level is labelled above the corresponding bar. (B) The vigilance (left) and memory (right) decrements binned by COVID-I 9 symptom severity. Each grey dot represents individual data and the error bar indicates I SE. An LMM with participant as a random effect showed that COVID-19 severity level had main effect on vigilance decrement $[F(I, 8)=6.8, P=0.03]$ and main effect on memory decmrenet $[F(I, 6)=15.3, P=0.008]$. Similarly, (D) shows the cognitive decrements for each long-COVID symptom severity level. An LMM with participant as a random effect showed that long-COVID symptoms had no effect on vigilance decrement $[F(I, 8)=4.5, P=0.07]$ or memory decrement $[F(I, 6)=0.09, P=0.8]$.

socioeconomic measures, encompassing education, income, occupation, work sector as well as subjective SES measured by the MacArthur Scale of Subjective Social Status. ${ }^{33}$ One hundred and seventeen responded $(75 \%$ of 155 participants, COVID $N=51$, control $N=66$ ), with no statistical difference in education level (Fig. 6F), annual income (Fig. 6G) or employment status (Fig. 6H). In fact, the COVID group reported a slightly higher subjective SES [Fig. 6I, COVID subjective $\mathrm{SES}=6.1$ (1.2), control subjective $\mathrm{SES}=5.6$ (1.5), COVID versus control: $t(96)=2.5, \quad P=0.02]$. Moreover, we found no difference in the proportion of essential workers amongst the groups (Fig. $6 \mathrm{~J}, \chi^{2}(1, N=51)$ $=0.8, P=0.4)$ or the method of the commute during the pandemic (Fig. 7A, all categories $P>0.1$ ). These suggest that SES cannot fully explain the cognitive difference between COVID and control groups in the present study. Another potential confound is testing experience; participants from the COVID group might, by chance, have less experience of cognitive testing than the controls. This hypothesis can be tested by comparing the number of studies that the participants attended on Prolific. In contrast, the COVID group had greater experience of online experiments $\left[t(134)=4.4, P<10^{-4}\right]$. The average number of studies attended was 248 studies (SD 358) in the COVID group and 67 (SD 82) in the control group. Moreover, the number of attended studies does not correlate with vigilance decrement (Spearman's $\rho=-0.09, P=0.3$ ) or memory decrement (Spearman's $\rho=-0.05, P=0.7$ ). These confirm that cognitive differences observed in the present study were not caused by the effect of test familiarity. Although it is unclear if smoking history could affect the cognitive ability, we also checked for this. Reassuringly, there was no difference in the proportion of past smokers [Fig. 7B, $\chi^{2}(1, N=29)=$ $0.9, P=0.3$ ] or present smokers [Fig. $7 \mathrm{C}, \chi^{2}(1, N=14)=$ $0.9, P=0.4]$ between the two groups. Finally, we asked for COVID-19 vaccination history: unsurprisingly, there 
$\operatorname{COVID}(n=53)$

Control $(n=83)$
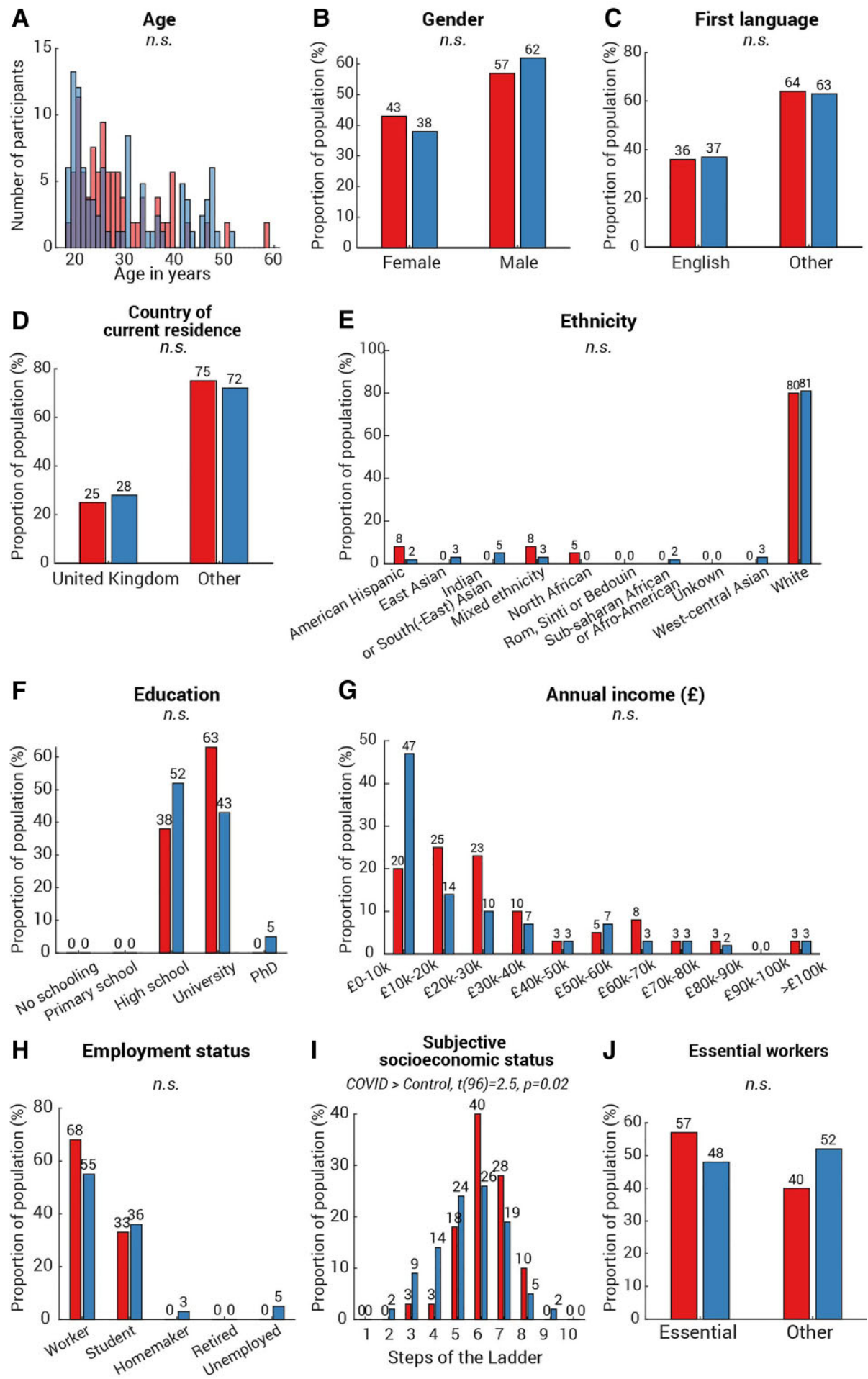

Figure 6 Demographics and socioeconomics profile of the participants. $T$-test was used to assess between-group difference in age (A) and subjective SES (I). For the measures with binary outcomes, including gender (B), first language (C), country of current residence (D), and essential workers $(\mathbf{J}), X^{2}$-test was used to assess between-group differences. Their $P$-values were unadjusted for multiple comparison. For the measures with multiple categories - ethnicity $(\mathbf{E})$, education $(\mathbf{F})$, annual income $(\mathbf{G})$ and employment status $(\mathbf{H}), X^{2}$-test was run for each category of each measure and $P$-values were adjusted using the Bonferroni method (i.e. multiplying the number of categories in that measure). Amongst all measures, only one measure showed significant difference: the COVID group showed a significantly higher subjective $S E S[H, t(96)=$ $2.5, P=0.02]$. No difference was found in other measures and annotated as n.s. (not significant). 

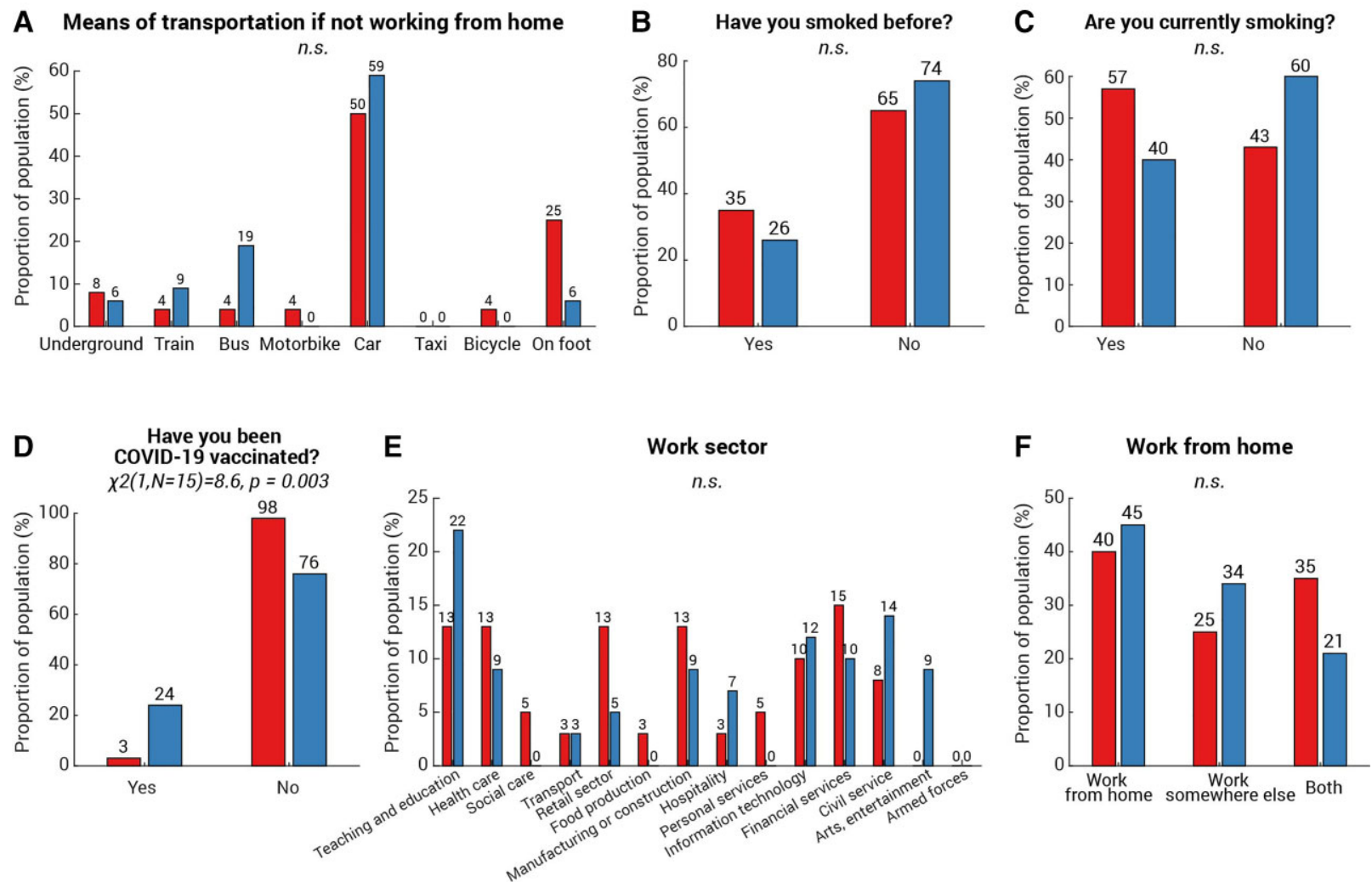

Figure 7 Work, smoking history and vaccination status of the participants. For the measures with multiple categories-transport means used to commute $(\mathbf{A})$, work sector $(\mathbf{E})$ and work from home status $(\mathbf{F}), X^{2}$-test was run for each category of each measure and $P$-values were adjusted using the Bonferroni method (i.e. multiplying the number of categories in that measure). For the measures with binary outcomes, including smoking history (B and C) and COVID-19 vaccination history (D), $X^{2}$-test was used to assess between-group differences. Amongst all measures, only one measure showed significant difference: the COVID group showed a lower rate of COVID-I9 vaccination $\left[D, X^{2}(I, N=I 5)=\right.$ 8.6, $P=0.003$ ]. No difference was found in other measures and annotated as n.s. (not significant).

was a significantly higher vaccination rate amongst controls than COVID-19 survivors [Fig. $7 \mathrm{D}, \chi^{2}(1, N=15)=8.6, P=$ $0.003]$. On average, the days from the last dose of vaccination to the date of attending the test was 63.7 (SD 37.9, minimum $=12$, maximum $=139$ ) and was not correlated with the vigilance decrement (Spearman's $\rho=0.1, P=0.6$ ). Taking all the evidence together, the results suggest that the cognitive differences in attention and memory observed here seem to be strongly associated with COVID-19 infection, rather than an outcome of a single demographic or socioeconomic metric.

Encouragingly, apart from these two cognitive differences, COVID-19 survivors did not show any significant difference from the age-matched controls in a wide range of cognitive capabilities, including short-term memory (Object Memory, Word Memory, Spatial Span), the response speed (Simple Reaction Task, Motor Control, Choice Reaction Time), spatial-visual attention (Target Detection), spatial planning (Tower of London), semantic reasoning (Verbal Analogies) and mental rotations (2D or 3D) (see Tables 3 and 4 for details). Similarly, comparing
PCA scores between COVID-19 survivors and controls, no significant difference was found in the short-term memory $[t(78)=-1.2, P=0.2, \mathrm{BF}=2.2]$, the executive function $[t(78)=0.3, P=0.7, \mathrm{BF}=4.1]$ or the mental rotation ability $[t(73)=0.2, P=0.8, \mathrm{BF}=4.2]$, suggesting that most of the key cognitive functions were normal.

\section{Discussion}

In the present study, we examined a wide range of cognitive abilities in COVID-19 survivors and age-matched controls. The COVID group did not require hospitalization and had not sought medical help for long-COVID symptoms after recovery. The good news is that COVID-19 survivors performed well in most cognitive abilities tested, including working memory, executive function, planning and mental rotation. However, even though their questionnaire-derived measures (fatigue, forgetfulness, motivation, sleep abnormality, depression and anxiety levels) were no different from age-matched controls (Table 1), they showed a 
significantly larger vigilance decrement along with faster fatigue build-up over the course of a 9 min-long attentionally demanding task (Fig. 3A and C). They also had significantly worse episodic memory decrement over time, comparable to a healthy, elderly person in their 60s (Fig. 4B). Notably, both deficits scaled with the time from COVID-19 diagnosis suggesting a strong relation with COVID-19 itself (Figs 3E and 4D).

To our knowledge, this is the first report describing deficits in sustained attention and episodic memory amongst mildly-affected COVID-19 survivors long after the acute illness, in people who were not complaining of long-COVID symptoms (cf. Zhou et al. ${ }^{9}$ reporting sustained attention impairments in recently-recovered patients). Our findings are consistent with the most prevalent complaints concerning post-COVID cognitive issues, including poor concentration and/or impaired memory in $18-50 \%$ of patients postrecovery. ${ }^{8,34,35}$ However, in contrast with previous reports of long-COVID patients, ${ }^{6-8,12,13,16}$ here we found no difference in short-term attention (performance over the first few minutes of a vigilance test; overall performance in tasks measuring executive function and response speed, Table 4) or working memory (performance in the immediate object or word memory tests, Tables 3 and 4 and Fig. 4A).

In the present study, COVID-19 survivors began with apparently normal behavioural performance followed by a gradual decline away from age-matched controls, suggesting reduced ability to attentively track and maintain information over time. The inconsistency with previous reports might be due to the fact that patients featured in those studies had severe COVID-19 symptoms, ${ }^{3,4,6,12}$ clinically significant cognitive impairment ${ }^{16}$ or at least reported persistent cognitive symptoms, ${ }^{7,8,13}$ whilst our participants were mostly nonhospitalized and devoid of self-reported abnormality.

The mechanisms underlying these cognitive deficits as yet remains unclear. Although a direct effect of virus persisting in the brain cannot be excluded, the evidence from postmortem studies suggests there is very little presence of virus within the brain in COVID-19 patients. ${ }^{36}$ Rather, there might be indirect effects of the virus on cognitive function mediated via a range of possible mechanisms, including immunological and microvascular changes (see review ${ }^{37}$ ). One investigation of COVID-19 survivors demonstrated that the most severely cognitively affected patients demonstrated a degree of cognitive impairment accompanied by hypometabolism in the frontoparietal regions. ${ }^{10}$ These brain regions are implicated in sustained attention ${ }^{38}$ as well as in episodic memory. ${ }^{39-41}$ Reassuringly, the follow-up study of Hosp et al. ${ }^{10}$ showed slow but evident improvement after 6 months. ${ }^{11}$ This is in line with the mildly-affected individuals reported here: both vigilance and episodic memory decrements gradually resolved over time (Figs $3 \mathrm{E}$ and $4 \mathrm{C}$ ). Episodic memory returned to normal levels after 6 months (Fig. 4D) and those who had COVID-19 over 9 months ago did not exhibit the vigilance decrement (Fig. 3F).

Unlike other survey-based reports focusing on selfreported long-COVID symptoms, ${ }^{35,42-44}$ COVID-19 survivors in the present study did neither indicated any sign of higher fatigue, forgetfulness, apathy, anxiety, depression or sleep abnormality (Table 1), nor felt any more tired than their age-matched controls over the time course of the vigilance test, suggesting a dissociation between self-report symptoms and objectively measured deficits. Our findings highlight that cognitive reductions are not limited to patients who had prolonged neurological manifestations after recovery, ${ }^{7,8}$ but might exist more widely in a sub-clinical form amongst COVID-19 survivors who would not consider themselves requiring any post-COVID treatment.

At the outset of the pandemic, Hampshire et al. ${ }^{17}$ conducted a large-scale online test involving over 13000 people with suspected or biologically confirmed COVID-19 circa 2 months. That study shared a subset of tasks with our investigation, covering a wide range of cognitive functions including semantic problem solving, visual-spatial attention, speed of response and working memory. All functions showed some degree of cognitive deficit amongst people who had contracted COVID-19, scaling with respiratory symptom severity. In the present study, however, we did not find any group differences in these cognitive domains (Table 4). This difference is likely to be attributable to the relative mildness of the symptoms experienced by our COVID-19 survivors, combined with the length of time since infection (some over 9 months ago). This suggests that these functional deficits might not be obvious in milder COVID-19 patients, with recovery expected within months.

There are some limitations to our study. Although the majority of participants from our COVID group reported a PCR-confirmed COVID-19 positive result with none reporting the need for post-COVID treatment, our study was limited by our reliance on self-reports of positive/negative COVID-19 tests and timing of diagnosis, which might increase or decrease our estimate of the prevalence and duration of COVID-associated cognitive deficits. Our study is also constrained by a relatively small sample size $(N=$ 136) with under-representation of the over 70s, thus any generalization should be taken carefully.

Overall, the findings here show that COVID-19 survivors showed a significant reduction in their ability to sustain attention on a demanding task up to 9 months after COVID-19 infection, along with mild, but significantly worse, episodic memory for up to 6 months. Just as the acute illness of COVID-19 demonstrates a wide severity spectrum from asymptomatic to fatal forms, ${ }^{45}$ our findings show that post-COVID cognitive deficits too can also manifest a wide severity spectrum. They highlight a pressing need to measure cognitive performance objectively in order to better understand how the brain is affected by COVID-19.

\section{Acknowledgements}

The authors are grateful to Stephen Walsh for his involvement in the early stage of the project. 


\section{Funding}

This work was supported by the Wellcome Trust and National Institute for Health Research (NIHR) Oxford Biomedical Research Centre. S.Z. and M.H. were funded by the Wellcome Trust (206330/Z/17/Z). S.M. was funded by an Medical Research Council (MRC) Clinician Scientist Fellowship (MR/P00878/X). K.S. was funded by the Berrow Foundation. A.H. was supported by the UK Dementia Research Institute Care Research, NIHR Technology Centre and Imperial Biomedical Research Centre. P.J.H. was supported by the NIHR Biomedical Research Centre, South London and Maudsley NHS Foundation Trust and King's College London. W.T. was funded by the Engineering and Physical Sciences Research Council (EPSRC) through the Imperial College Centre for Doctoral Training (CDT) for Neurotechnology. The funders had no role in study design, data collection and analysis, decision to publish or preparation of the manuscript.

\section{Competing interests}

The authors declare no competing financial interests.

\section{References}

1. Taquet M, Geddes JR, Husain M, Luciano S, Harrison PJ. 6-month neurological and psychiatric outcomes in 236379 survivors of COVID-19: A retrospective cohort study using electronic health records. Lancet Psychiatry 2021;8(5):416-427.

2. Mao L, Jin H, Wang M, et al. Neurologic manifestations of hospitalized patients with coronavirus disease 2019 in Wuhan, China. JAMA Neurol. 2020;77(6):683-690.

3. Helms J, Kremer S, Merdji $\mathrm{H}$, et al. Neurologic features in severe SARS-CoV-2 infection. N Engl J Med. 2020;382(23): $2268-2270$.

4. Varatharaj A, Thomas N, Ellul MA, et al. Neurological and neuropsychiatric complications of COVID-19 in 153 patients: A UK-wide surveillance study. Lancet Psychiatry. 2020;7(10): 875-882.

5. Nalbandian A, Sehgal K, Gupta A, et al. Post-acute COVID-19 syndrome. Nat Med. 2021;27(4):601-615.

6. Jaywant A, Vanderlind WM, Alexopoulos GS, Fridman CB, Perlis $\mathrm{RH}$, Gunning FM. Frequency and profile of objective cognitive deficits in hospitalized patients recovering from COVID-19. Neuropsychopharmacology. 2021;46:2235-2240.

7. Graham EL, Clark JR, Orban ZS, et al. Persistent neurologic symptoms and cognitive dysfunction in non-hospitalized Covid-19 "long haulers". Ann Clin Transl Neurol. 2021;8(5):1073-1085.

8. Woo MS, Malsy J, Pöttgen J, et al. Frequent neurocognitive deficits after recovery from mild COVID-19. Brain Commun. 2020;2(2): fcaa205.

9. Zhou H, Lu S, Chen J, et al. The landscape of cognitive function in recovered COVID-19 patients. J Psychiatr Res. 2020;129:98-102.

10. Hosp JA, Dressing A, Blazhenets G, et al. Cognitive impairment and altered cerebral glucose metabolism in the subacute stage of COVID-19. Brain. 2021;144(4):1263-1276.

11. Blazhenets G, Schröter N, Bormann T, et al. Slow but evident recovery from neocortical dysfunction and cognitive impairment in a series of chronic COVID-19 patients. J Nucl Med. 2021;62: 910-915.
12. Beaud V, Crottaz-Herbette S, Dunet V, et al. Pattern of cognitive deficits in severe COVID-19. J Neurol Neurosurg Psychiatry. 2021;92:567-568.

13. Hellmuth J, Barnett TA, Asken BM, et al. Persistent COVID-19-associated neurocognitive symptoms in nonhospitalized patients. J Neurovirol. 2021;27(1):191-195.

14. Raman B, Cassar MP, Tunnicliffe EM, et al. Medium-term effects of SARS-CoV-2 infection on multiple vital organs, exercise capacity, cognition, quality of life and mental health, post-hospital discharge. EClinicalMedicine. 2021;31:100683.

15. Ferrucci R, Dini M, Groppo E, et al. Long-lasting cognitive abnormalities after COVID-19. Brain Sci. 2021;11(2):235.

16. Miskowiak K, Johnsen S, Sattler SM, et al. Cognitive impairments four months after COVID-19 hospital discharge: Pattern, severity and association with illness variables. Eur Neuropsychopharmacol. 2021;46:39-48.

17. Hampshire A, Trender W, Chamberlain SR, et al. Cognitive deficits in people who have recovered from COVID-19. EClinicalMedicine. 2021;39:101044.

18. Mills RJ, Young CA, Pallant JF, Tennant A. Development of a patient reported outcome scale for fatigue in multiple sclerosis: the Neurological Fatigue Index (NFI-MS). Health Qual Life Outcomes. 2010;8(1):22.

19. Broadbent DE, Cooper PF, FitzGerald P, Parkes KR. The Cognitive Failures Questionnaire (CFQ) and its correlates. Br J Clin Psychol. 1982;21(1):1-16.

20. Lang FR, John D, Lüdtke O, Schupp J, Wagner GG. Short assessment of the Big Five: robust across survey methods except telephone interviewing. Behav Res Methods. 2011;43(2):548-567.

21. Duckworth AL, Quinn PD. Development and validation of the Short Grit Scale (Grit-S). J Pers Assess. 2009;91:166-174.

22. Ang Y-S, Lockwood P, Apps MAJ, Muhammed K, Husain M. Distinct subtypes of apathy revealed by the apathy motivation index. PLoS ONE. 2017;12(1):e0169938.

23. Zigmond AS, Snaith RP. The hospital anxiety and depression scale. Acta Psychiatr Scand. 1983;67(6):361-370.

24. Nuechterlein KH, Parasuraman R, Jiang Q. Visual sustained attention: image degradation produces rapid sensitivity decrement over time. Science. 1983;220(4594):327-329.

25. Peirce J, Gray JR, Simpson S, et al. PsychoPy2: Experiments in behavior made easy. Behav Res Methods. 2019;51(1):195-203.

26. Efron B, Tibshirani RJ. An introduction to the bootstrap. CRC Press; 1994.

27. Corsi PM. Human memory and the medial temporal region of the brain. Unpublished PhD thesis. McGill University; 1972.

28. Berch DB, Krikorian R, Huha EM. The Corsi block-tapping task: Methodological and theoretical considerations. Brain Cogn. 1998;38(3):317-338.

29. Shallice T, Broadbent DE, Weiskrantz L. Specific impairments of planning. Philos Trans R Soc Lond B Biol Sci. 1982;298(1089): 199-209.

30. Silverman I, Choi J, Mackewn A, Fisher M, Moro J, Olshansky E. Evolved mechanisms underlying wayfinding. further studies on the hunter-gatherer theory of spatial sex differences. Evol Hum Behav. 2000;21(3):201-213.

31. Mena GE, Martinez PP, Mahmud AS, Marquet PA, Buckee CO, Santillana M. Socioeconomic status determines COVID-19 incidence and related mortality in Santiago, Chile. Science. 2021;372(6545): eabg5298.

32. Hackman DA, Farah MJ, Meaney MJ. Socioeconomic status and the brain: mechanistic insights from human and animal research. Nat Rev Neurosci. 2010;11(9):651-659.

33. Adler N, Stewart J. The MacArthur scale of subjective social status. San Francisco: MacArthur Research Network on SES \& Health. 2007.

34. Rogers JP, Chesney E, Oliver D, et al. Psychiatric and neuropsychiatric presentations associated with severe coronavirus infections: a systematic review and meta-analysis with comparison to the COVID-19 pandemic. Lancet Psychiatry. 2020;7(7):611-627. 
35. Blomberg B, Mohn KG-I, Brokstad KA, et al. Long COVID in a prospective cohort of home-isolated patients. Nat Med. 2021;27: 1607-1613.

36. Lee M-H, Perl DP, Nair G, et al. Microvascular injury in the brains of patients with Covid-19. N Engl J Med. 2021;384(5):481-483.

37. Alnefeesi Y, Siegel A, Lui LMW, et al. Impact of SARS-CoV-2 infection on cognitive function: A systematic review. Front Psychiatry. 2021;11:621773. doi:10.3389/fpsyt.2020.621773

38. Langner R, Eickhoff SB. Sustaining attention to simple tasks: a meta-analytic review of the neural mechanisms of vigilant attention. Psychol Bull. 2013;139(4):870-900.

39. Cabeza R, Ciaramelli E, Olson IR, Moscovitch M. The parietal cortex and episodic memory: An attentional account. Nat Rev Neurosci. 2008;9(8):613-625.

40. Davis SW, Wing EA, Cabeza R. Contributions of the ventral parietal cortex to declarative memory. Handb Clin Neurol. 2018;151: $525-553$.
41. Dudukovic NM, Kuhl BA. Cognitive control in memory encoding and retrieval. In: The Wiley handbook of cognitive control. John Wiley \& Sons, Ltd; 2017:355-375.

42. Carfi A, Bernabei R, Landi F, for the Gemelli Against COVID-19 Post-Acute Care Study Group. Persistent symptoms in patients after acute COVID-19. JAMA. 2020;324:603-605.

43. Huang C, Huang L, Wang Y, et al. 6-month consequences of COVID-19 in patients discharged from hospital: a cohort study. Lancet. 2021;397:220-232.

44. Cirulli ET, Barrett KMS, Riffle S, et al. Long-term COVID-19 symptoms in a large unselected population. medRxiv. Published online December 1, 2020:2020.10.07.20208702. 10.1101/2020.10. 07.20208702

45. Wiersinga WJ, Rhodes A, Cheng AC, Peacock SJ, Prescott HC. Pathophysiology, transmission, diagnosis, and treatment of coronavirus disease 2019 (COVID-19): A review. JAMA. 2020;324(8): 782-793. 